A. M. Steinberg, I. Boxx, M. Stöhr, C. D. Carter, W. Meier, Flow-flame interactions causing acoustically coupled heat release fluctuations in a thermoacoustically unstable gas turbine model combustor, Combustion and Flame 157 (2010) 2250-2266.

The original publication is available at www.elsevier.com

http://dx.doi.org/10.1016/j.combustflame.2010.07.011 


\title{
Flow-flame interactions causing acoustically coupled heat release fluctuations in a thermo-acoustically unstable gas turbine model combustor
}

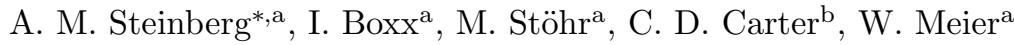 \\ ${ }^{a}$ Institute for Combustion Technology, German Aerospace Centre (DLR), 70569 Stuttgart, Germany \\ ${ }^{b}$ Air Force Research Laboratory, Wright-Patterson AFB, OH, USA
}

\begin{abstract}
A detailed analysis of the flow-flame interactions associated with acoustically coupled heat-release rate fluctuations was performed for a $10 \mathrm{~kW}, \mathrm{CH}_{4}$ /air, swirl stabilized flame in a gas turbine model combustor exhibiting self-excited thermoacoustic oscillations at $308 \mathrm{~Hz}$. High-speed stereoscopic particle image velocimetry, OH planar laser induced fluorescence, and $\mathrm{OH}^{*}$ chemiluminescence measurements were performed at a sustained repetition rate of $5 \mathrm{kHz}$, which was sufficient to resolve the relevant combustor dynamics. Using spatio-temporal proper orthogonal decomposition, it was found that the flow-field contained several simultaneous periodic motions: the reactant flux into the combustion chamber periodically oscillated at the thermo-acoustic frequency $(308 \mathrm{~Hz})$, a helical precessing vortex core $(\mathrm{PVC})$ circumscribed the burner nozzle at $515 \mathrm{~Hz}$, and the PVC underwent axial contraction and extension at the thermo-acoustic frequency. The global heat release rate fluctuated at the thermo-acoustic frequency, while the heat release centroid circumscribed the combustor at the difference between the acoustic and PVC frequencies. Hence, the three-dimensional location of the heat release fluctuations depended on the interaction of the PVC with the flame surface. This motivated the compilation of doubly phase resolved statistics based on the phase of both the acoustic and PVC cycles, which showed highly repeatable periodic flow-flame configurations. These include flames stabilized between the inflow and inner recirculation zone, large-scale flame wrap-up by the PVC, radial deflection of the inflow by the PVC, and combustion in the outer recirculation zones. Large oscillations in the flame surface area were observed at the thermo-accoustic frequency that significantly affected the total heat-release oscillations. By filtering the instantaneous reaction layers at different scales, the importance of the various flow-flame interactions affecting the flame area was determined. The greatest contributor was large-scale elongation of the reaction layers associated with the fluctuating reactant flow rate, which accounted for approximately $50 \%$ of the fluctuations. The remaining $50 \%$ was distributed between fine scale stochastic corrugation and large-scale corrugation due to the PVC.
\end{abstract}

Published by Elsevier, Combustion and Flame, 157 (2010) 2250-2266

Key words: Thermo-acoustic instabilities, gas turbine combustors, swirl stabilized flames, turbulence-flame interaction, high-speed diagnostics, proper orthogonal decomposition

\section{Introduction}

Lean swirl-stabilized flames are extensively employed in modern gas turbine combustors in order to reduce $\mathrm{NO}_{x}$ emissions [1-3]. However, combustion driven instabilities can occur in such systems when periodic fluctuations in the heat release rate couple with one of the acoustic modes of the combustion chamber. The ensuing feed-back loop adds energy to both the heat release and pressure fluctuations, increasing their amplitude. This can lead to unsteady operation, flame extinction, and mechanical damage to the engine. Considerable effort therefore has been made to better understand these thermo-acoustic instabilities and extensive reviews are provided in Refs. [4-7].

While various active and passive control mechanisms have been developed to mitigate thermo-acoustic insta-

*Corresponding author email: adam.steinberg@dlr.de bilities [8-11], there are still many unanswered questions regarding the fundamental mechanisms causing coupled acoustic and heat release fluctuations. Candel [4] identified eleven different flow and combustion processes that could lead to instabilities in different situations. However, understanding how and when these mechanisms couple with acoustic fluctuations is very difficult due to the complexity of the flow-flame interactions occurring in swirl-stabilized combustors. For example, a variety of studies have shown that swirl-induced vortex breakdown can lead to the formation of large-scale helical vortex structures that precess around the combustor and greatly affect the mixing and combustion [12-17].

The application of advanced laser-based diagnostics to gas-turbine relevant flames has provided considerable insight into these interactions and other phenomena important to thermo-acoustic instabilities. Such investigations have targeted systems ranging from simple, unconfined 
flames $[18,19]$ to high-temperature, high-pressure combustors using complex fuels [20-26]. However, the simple flame configurations are typically designed to study one particular fundamental process and do not describe the complex flow-flame interactions occurring in practical systems. Conversely, experiments at operating conditions similar to those of practical gas-turbines are unable to provide systematic and detailed investigations of these interactions due to uncertainty in boundary conditions, limited optical access, expense, and the reduced effectiveness of several important measurement techniques in highpressure and/or liquid fueled flames. The advancement of Large Eddy Simulations (LES), which resolve the integral time and length scale flow/flame dynamics, also have provided new insight into the behavior of swirl stabilized combustors [27-33]. However, such simulations require models for the turbulence, flame, and turbulence-flame interactions occurring in the subgrid, which are still a topic of research [7, 34-36].

It therefore is useful to investigate gas-turbine-like swirlflames in well controlled, rigorously defined, and highly repeatable laboratory conditions that are designed to allow for the application of advanced laser diagnostics. Such gas turbine model combustors (GTMC) exhibit many of the features of the practical systems, such as self-excited thermo-acoustic instabilities and precessing vortex cores, while remaining sufficiently manageable that experimental measurements can provide significant physical insight and detailed model validation data [37-49]. In the present study, a laboratory scale gas turbine combustor has been investigated under thermo-acoustically unstable conditions at atmospheric pressure. The burner is a modified version of a practical gas turbine swirl combustor in which fuel and air entered in separate streams, but partially premixed before combustion. The three-component planar velocity field, planar laser induced fluorescence (PLIF) from the $\mathrm{OH}$ combustion radical, and chemiluminescence from electronically excited $\mathrm{OH}\left(\mathrm{OH}^{*}\right)$ all were measured at a sustained repetition rate of $5 \mathrm{kHz}$, allowing resolution of the thermo-acoustic combustor dynamics.

This combustor has been the subject of numerous previous experimental investigations. Giezendanner et al. [37, 38 ] studied a lean methane-air flame that exhibited selfexcited thermo-acoustic oscillations in the range of 290 $\mathrm{Hz}$ using $\mathrm{OH}, \mathrm{CH}$, and $\mathrm{CH}_{2} \mathrm{O}$ PLIF, laser Doppler velocimetry (LDV), and $\mathrm{OH}^{*}$ chemiluminescence. The PLIF and chemiluminescence measurements were performed at a rate of $10 \mathrm{~Hz}$ and locked to various phase angles of the dominant acoustic oscillation. Duan et al. [39] studied the same flame using phase-resolved LDV, Raman scattering, and $\mathrm{OH} / \mathrm{CH}$ PLIF. It was found that both the fuel and air flow rates underwent significant variations over an acoustic cycle. Furthermore, there was a significant correlation between the mass flux (measured from the axial velocity at the nozzle exit) and the heat release rate in the combustion chamber. Using similar diagnostics, Weigand et al. [40] showed that there were periodic variations in the mixing of hot products with fresh reactants. This could cause ignition of such reactants and increase the global heat release rate.

Recent work in this combustor has focused on flowflame interaction, with the aim of better understanding the mechanisms that drive the instability. Weigand et al. [41] and Meier et al. [42] investigated three different GTMC flames using $\mathrm{OH} / \mathrm{CH}$ PLIF, LDV, and Raman scattering. The flames studied demonstrated a wide range of behaviors, with one exhibiting self-excited thermo-acoustic oscillations, one burning stably, and one exhibiting periodic lean blowout. In all cases, it was found that the flame did not stabilize directly on the fuel nozzle, but was lifted by several millimeters. This allowed the fuel and air to partially premix before combustion began at the flame root. The combustion behavior in the near-field of the nozzle was influenced both by mixing and finite rate chemistry. Reactions occurred in thin layers and strong turbulencechemistry interactions were observed to cause local extinction of these layers.

Such interactions were further elucidated by Stöhr et al. [43, 45] and Sadanandan et al. [44] using stereoscopic particle image velocimetry (PIV) and OH PLIF. By applying the method of proper orthogonal decomposition (POD), it was shown that the dominant flow structure was a helical vortex that precessed around the burner nozzle at a frequency that was independent of the acoustics. Considerable mutual interaction between this precessing vortex core (PVC) and flame was observed. Most recently, Boxx et al.[50] studied this combustor using highrepetition-rate laser and optical diagnostics. Stereoscopic PIV, OH PLIF, and $\mathrm{OH}^{*}$ chemiluminescence all were applied at a sustained repetition rate of $5 \mathrm{kHz}$. This allowed qualitative observation of several important flow-flame interactions including flame roll up, local extinction, and possible auto-ignition events. Frequency analysis of the PVC was conducted using both POD analysis and monitor points at characteristic locations in the combustor.

The wealth of experimental data has yielded considerable insight into both the behavior of this burner and important processes for swirl stabilized combustion in general. However, a complete description of the flow-field dynamics and, in particular, how they couple with heat release oscillations has remained elusive. Low repetitionrate (i.e. $10 \mathrm{~Hz}$ ) measurements can be correlated with a particular phase of the acoustic cycle to determine phasecorrelated statistics. However, deriving phenomenological descriptions from such an analysis requires the flow and flame have the same statistics at the measurement location over every acoustic cycle, which does not occur due to the three-dimensional, asymmetric PVC. In fact, due to its motion around the combustion chamber, phase-correlated velocity statistics at the thermo-acoustic frequency may not capture the PVC at all [41]. Furthermore, instantaneous measurements at the same phase of the acoustic cycle will indicate very different phenomenology depending on the three-dimensional position of the PVC relative 
to the measurement location.

To overcome the limitations of conventional $10 \mathrm{~Hz}$ measurement techniques, high-repetition-rate (multi-kHz) planar laser diagnostics recently have been applied to a variety of turbulence and combustion problems. In configurations with low through-plane motion, such diagnostics have the capability of explicitly resolving important dynamic processes [51-57]. High-repetition-rate diagnostics also have been applied to study swirl-stabilized flames in gas-turbine-like combustors $[50,58]$. Previous work in this burner using such diagnostics has investigated individual time sequences of OH PLIF and stereoscopic PIV measurements in order to observe the dynamics of the flame and flow [50]. However, the high through-plane motion in these flames means that subsequent measurements are not well correlated in time; the measurements at a given time are not the continuation of the same fluid and flame that was measured at the previous time. It therefore is not possible to determine if the observed events are due to transient interactions or through-plane convection of quasi-stable configurations. Furthermore, the results in Ref. [50] show that individual time sequences appear to have a very chaotic behavior. While such issues may be mitigated by using multiple simultaneous measurement planes, it is not currently feasible to characterize the full three-dimensional, temporally evolving flow-field and flame.

In contrast to previous investigations of individual time sequences, this work focuses on using characteristic spatial and temporal signals from planar high-speed measurements to investigate repeatable phenomena that are associated with the thermo-acoustic instability. That is, the important flow-flame interactions for driving a thermoacoustic instability are, by necessity, periodic over the acoustic cycle somewhere in the combustor. Correct interpretation of these interactions therefore requires simultaneous consideration of the acoustics and the three-dimensional asymmetry brought about by the PVC. The analysis of these interactions in this work follows several steps. First, important periodic dynamics of the flow-field are described based on the high-speed velocity measurements. The highspeed chemiluminescence and PLIF measurements are then used to qualitatively describe important, large-scale features of the flow-flame interaction, which were strongly influenced by the dynamics of the PVC. It then is shown that the high-speed measurements can be used to compile statistics that are simultaneously resolved with respect to the phase angles of the acoustic cycle and the PVC motion around the combustion chamber. These statistics reveal flow-field and flame configurations that are very repeatable when both phase angles are considered together. Finally, the insights gained from the doubly phase resolved statistics are combined with the temporal measurements to describe the flow-flame interactions causing acoustically coupled heat release fluctuations.

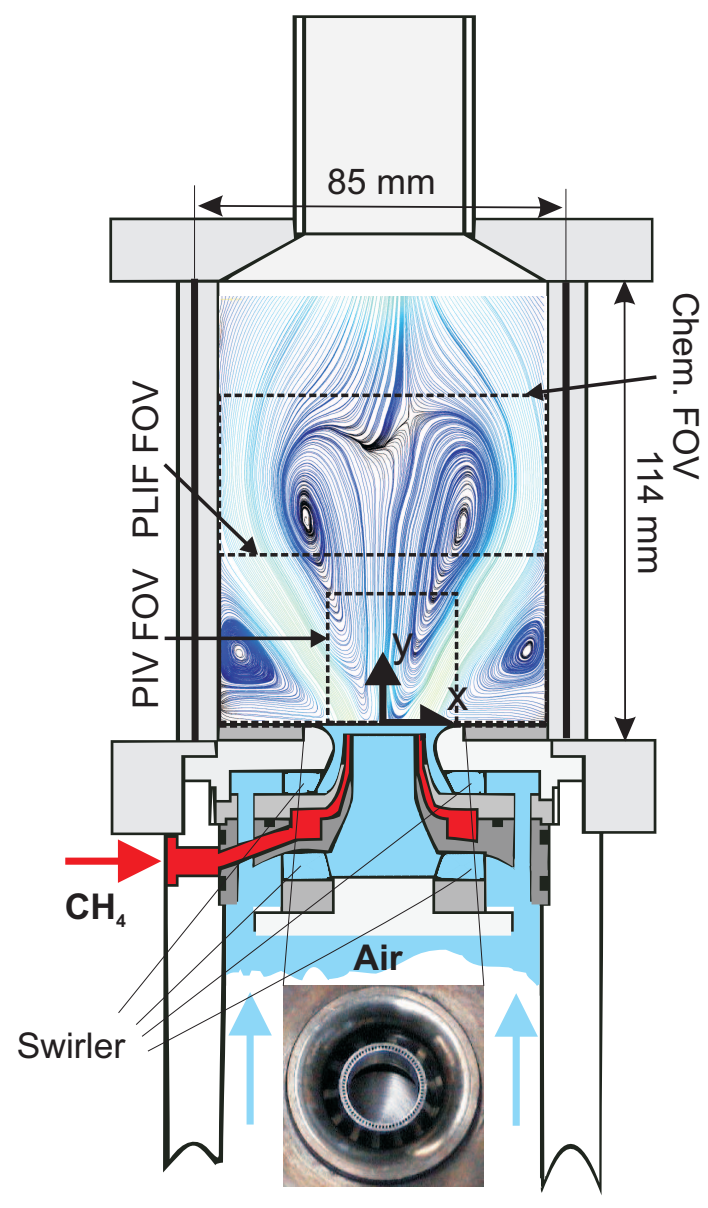

Figure 1: Schematic of the gas turbine model combustor with the fields of view for the various diagnostics. The streamlines indicate the mean velocity-field measured in Ref. [44].

\section{Combustor and diagnostics}

The GTMC used for this work has been the subject of numerous previous studies in which the geometry is described in detail [37-45]. Furthermore, the analysis presented in this work is based on the experiments of Ref. [50]. Hence, only a brief description of the combustor and diagnostics is provided here.

\subsection{Gas turbine model combustor}

The GTMC is shown schematically in Fig. 1. Coswirling dry air at room temperature was supplied to the flame through a central nozzle (diameter $15 \mathrm{~mm}$, eight swirl channels) and an annular nozzle (i.d. $17 \mathrm{~mm}$, o.d. $25 \mathrm{~mm}$ contoured to an o.d. of $40 \mathrm{~mm}, 12$ swirl channels), both of which were fed from a common plenum. Nonswirling $\mathrm{CH}_{4}$ was introduced through a ring comprised of 72 square $0.5 \mathrm{~mm} \times 0.5 \mathrm{~mm}$ channels between the air nozzles. The exit plane of the fuel and central air nozzles was $4.5 \mathrm{~mm}$ below that of the outer nozzle and the latter was taken as the reference height $(y=0)$. 
The burner was enclosed in an optically accessible combustion chamber comprised of fused silica plates held in the corners by Inconel@ alloy posts. This allowed virtually unobstructed optical access to the flame. The chamber had a square cross section of $85 \mathrm{~mm} \times 85 \mathrm{~mm}$ and a height of $114 \mathrm{~mm}$. The exhaust was formed from a steel plate with a conical contraction, leading to a $40 \mathrm{~mm}$ diameter exhaust tube.

Under certain operating conditions, flames in this burner exhibit self-excited thermo-acoustic pulsations, while under other conditions the flames operate stably [41, 42]. The flame studied here corresponded to 'Flame B' in Refs. [41, 42], was identical to that studied in Ref. [50], and produced large-amplitude thermo-acoustic pulsations at 308 $\mathrm{Hz}$. Fuel and air were supplied to the combustion chamber at rates of $12.3 \mathrm{~g} / \mathrm{min}$ and $281 \mathrm{~g} / \mathrm{min}$, respectively. Flow rates were controlled using electromechanical mass flow controllers (Brooks) and monitored using calibration standard Coriolis mass flow meters (Siemens Sitrans F C) with an uncertainty of $1.5 \%$. The resultant flame had a thermal power of $10 \mathrm{~kW}$, a global equivalence ratio of 0.75 , and a swirl number of 0.55 . The Reynolds number based on the total reactant flow rate and the minimum outer nozzle diameter $(25 \mathrm{~mm})$ was about 15,000 . The burner was allowed to thermally stabilize at full power for at least 20 minutes prior to data acquisition. During a data acquisition run, approximately $4 \%$ of the air mass flow was diverted through a fluidized bed particle seeder containing $0.5 \mu \mathrm{m} \mathrm{TiO}_{2}$ particles to enable the velocity measurments. The air flow was seeded only during the short data acquisition periods to reduce the accumulation of particles on the windows. Becuase the flow only was seeded for a few seconds at a time, window degradation was minimized and several data acquisition runs could be accomplished before window contamination significantly affected the measurements.

The burner was equipped with multiple ports for microphone probes and pressure transducers. Two probes (Brüel \& Kjaer, Type 4939) were used for this study, one mounted in a corner post of the combustion chamber and one in the outer wall of the plenum. Both probes were sampled at a rate of $20 \mathrm{kHz}$ using a multichannel DAQ system, which simultaneously recorded the camera intensifier trigger for the PLIF system described below. This allowed synchronization of the acoustic and laser based measurements. The frequency spectra measured from the probes were virtually identical and showed a large amplitude acoustic fluctuation at $308 \mathrm{~Hz}$. The signal from the plenum microphone exhibited less noise than that from combustion chamber and had a phase shift of approximately $85^{\circ}$ in the dominant Fourier transformation frequency. Due to the reduced noise, this plenum signal was shifted in phase to match that in the combustion chamber and used to identify the phase angle in the acoustic cycle at which each laser based measurement was acquired. Examples of the acoustic signals are provided in Section 3 .

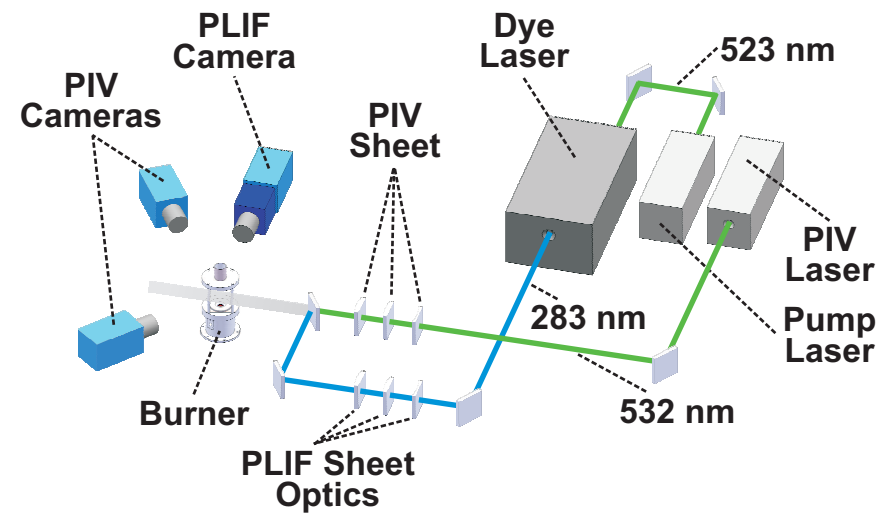

Figure 2: Layout of simultaneous $5 \mathrm{kHz}$ stereoscopic PIV and $\mathrm{OH}$ PLIF system.

\subsection{Stereoscopic particle image velocimetry}

Three-component planar velocity-fields were measured at a rate of $5 \mathrm{kHz}$ using stereoscopic PIV. The system, shown in Fig. 2, consisted of a high-repetition-rate, dualcavity, diode-pumped, solid state Nd:YAG laser (Edgewave, IS-6IIDE) and a pair of high-speed CMOS cameras (LaVision HSS5). Laser pulse pairs (532 nm, $2.6 \mathrm{~mJ} /$ pulse, $14 \mathrm{~ns}$ pulse duration, $20 \mu \mathrm{s}$ between pulses) repeating at $5 \mathrm{kHz}$ were expanded into a collimated sheet using a two component cylindrical lens telescope. The sheet was then focused to a width of $0.7 \mathrm{~mm}$ at the burner axis using a third cylindrical lens. Particle-scattered light from the $\mathrm{TiO}_{2}$ seed was collected into the cameras using $100 \mathrm{~mm}$ focal length commercial camera lenses at f/5.6 (Tokina).

The pair of CMOS cameras were mounted equidistant from opposite sides of the laser sheet in a forward-scatter configuration. Image defocussing due to the angular configuration was corrected by rotating the camera body with respect to the lens as stipulated by the Scheimpflug criterion. Perspective distortion brought about by this configuration was corrected by imaging a three-dimensional dot target (LaVision type 7) that was placed in the measurement plane. The distorted dot target was transformed to a normal coordinate system using the LaVision DaVis 7.2 software package. The same target images were used to align the fields-of-view from the PIV cameras with that from the PLIF camera described below.

Vector fields were computed from the particle image spatial cross-correlation using the LaVision DaVis 7.2 software package. An adaptive multi-pass vector evaluation technique was used, with interrogation boxes ranging from 64 pixels to 16 pixels. In dual frame mode at $5 \mathrm{kHz}$, the PIV cameras had an active sensor size of 512 x 512 pixels. The field-of-view imaged by the PIV system was 32 mm x $30 \mathrm{~mm}$ (shown in Fig. 1), resulting in a spatial resolution and vector spacing of approximately $0.94 \mathrm{~mm}$ and $0.47 \mathrm{~mm}$ respectively. Each camera had 2.6 GB of memory, allowing 4096 image pairs to be obtained over a measurement duration of approximately $0.8 \mathrm{~s}$. It is noted that the main advantage of the stereoscopic aspect of the 
diagnostics for the present analysis was to increase the accuracy of the computed in-plane velocity components. Stereoscopic PIV provides better accuracy than conventional one-camera PIV in flows with high through-plane motion due to reduced perspective error [59].

\subsection{Planar laser induced fluorescence and chemilumines- cence}

Laser induced fluorescence was used to measure the planar distribution of the $\mathrm{OH}$ combustion radical and was conducted simultaneously with the PIV measurements. The $\mathrm{OH}$ PLIF system consisted of a high-repetition-rate, frequency doubled Nd:YLF laser (Edgewave IS-8IIE) pumping a dye laser (Sirah Cobra-Stretch modified for $\mathrm{kHz}$ pumping). At $5 \mathrm{kHz}$, the pump laser delivered $3.8 \mathrm{~mJ} /$ pulse (19 W average output) at $523 \mathrm{~nm}$ with an $8.5 \mathrm{~ns}$ pulse duration. The output of the dye laser was frequency doubled and tuned to excite the $\mathrm{Q}_{1}(7)$ line of the A-X $(1,0)$ transition of $\mathrm{OH}$ at $283.2 \mathrm{~nm}$. Tuning of the laser wavelength was checked daily using a calibration burner. After frequency doubling, the average output power of the dye laser at $283.2 \mathrm{~nm}$ was $0.5 \mathrm{~W}$ or $0.1 \mathrm{~mJ} /$ pulse. The laser beam was formed into a sheet with a height of approximately $40 \mathrm{~mm}$ and a waist of approximately $0.4 \mathrm{~mm}$ using three cylindrical lenses. This sheet was overlapped with the PIV laser sheet using a dichroic mirror and sent into the test section along the same beam path.

Fluorescence of the $\mathrm{OH}$ radical in the range of 310 nm was acquired with a CMOS camera (LaVision HSS6) equipped with an external, two-stage, lens-coupled intensifier (LaVision HS-IRO) and a $45 \mathrm{~mm} \mathrm{f} / 1.8 \mathrm{UV}$ lens (Cerco). The intensifier gate time was set to $500 \mathrm{~ns}$ and the PLIF laser pulse occurred between the first and second PIV laser pulses on each cycle. The field-of-view imaged by the PLIF system covered the entire width of the combustion chamber $(85 \mathrm{~mm})$ and extended from the nozzle exit to a height of $40 \mathrm{~mm}$.

Background luminosity and elastic scattering were reduced by using a $500 \mathrm{~ns}$ intensifier gate, a high-transmission (> $80 \%$ at $310 \mathrm{~nm}$ ) bandpass interference filter (Laser Components $\mathrm{GmbH}$ ), and a color glass filter (1 mm thick WG295 Schott glass). The $\mathrm{OH}$ images were corrected for the mean laser sheet intensity profile, which was determined based on 1000 images of the fluorescence from a uniform acetone vapour that was doped into the test area. A correction also was made to remove the mean flame luminosity.

The line-of-sight integrated spontaneous emission of electronically excited $\mathrm{OH}\left(\mathrm{OH}^{*}\right)$ was imaged separately from the PLIF and PIV measurements, but under identical run conditions. The camera/lens/filter arrangement for the chemiluminescence camera was identical to that of the OH-PLIF camera; however, the intensifier gate time was extended to $25 \mu$ s in order to capture sufficient signal. The field-of-view was not restricted by a laser sheet height and chemiluminescence was collected over an $85 \times 85 \mathrm{~mm}$ region at a rate of $5 \mathrm{kHz}$ for a $0.8 \mathrm{~s}$ duration. The $\mathrm{OH}^{*}$ chemiluminescence was taken as a qualitative indicator of the integrated heat release rate in the combustor [8].

\section{Analysis of temporally resolved measurements}

To begin, important features of the combustor behavior will be described based on the temporally resolved measurements. These observations motivate the doubly phase resolved statistical analysis presented in Section 4. All images and statistics were extracted from two approximately $0.8 \mathrm{~s}$ long measurement sequences, containing a total of approximately 8000 simultaneous PIV/PLIF images.

\subsection{Flow-field dynamics}

For the present investigation using high-speed diagnostics, velocity measurements were not taken over the entire combustion chamber. However, such measurements were obtained using a conventional $10 \mathrm{~Hz}$ PIV system in Refs. [43-45]. The mean flow-field derived from these experiments is overlaid on the burner geometry in Fig. 1 and exhibited the large inner recirculation zone and smaller outer recirculation zones that are characteristic of swirl stabilized combustors. However, the same experiments also demonstrated that the instantaneous flow-fields did not resemble this mean configuration, but instead consisted of many individual vortices in a time varying configuration. Stöhr et al. [43] and Boxx et al. [50] showed that the turbulent structures with the highest kinetic energy represented a helical precessing vortex core (PVC) that circumscribed the burner nozzle at approximately $515 \mathrm{~Hz}$. The PVC has been observed under all operating conditions studied in this combustor (both reacting and nonreacting) and the precession frequency increased linearly with the mass flow rate. The PVC also is present in industrial gas turbines and has been shown to greatly affect thermo-acoustic instabilities (e.g. Ref. [13] and references therein).

Understanding the flow-flame interactions causing the thermo-acoustic instability requires understanding the repeatable flow-field dynamics in great detail. To achieve this, a spatio-temporal proper orthogonal decomposition (POD) analysis was applied to the high-speed velocity data. POD analysis is a mathematically rigorous method of extracting the most energetic features of a data set and has been successfully applied to the study of turbulence and combustion [29, 60-62]. Since the mathematical basis of POD is well established, only a brief description pertinent to this analysis is presented in Appendix A. In summary, POD analysis from a sequence of $n$ temporally resolved velocity-field measurements provides a set of $n$ spatial eigenmodes $\left(M_{j}(x, y)\right)$, temporal coefficients $\left(a_{j}(t)\right)$, and eigenvalues $\left(\lambda_{j}\right)$ such that the eigenmodes form an orthogonal basis for the original data set. The eigenvalues represent the contributions of the modes to the overall kinetic energy of the flow and the particular property of POD analysis is that it provides optimal convergence of the 


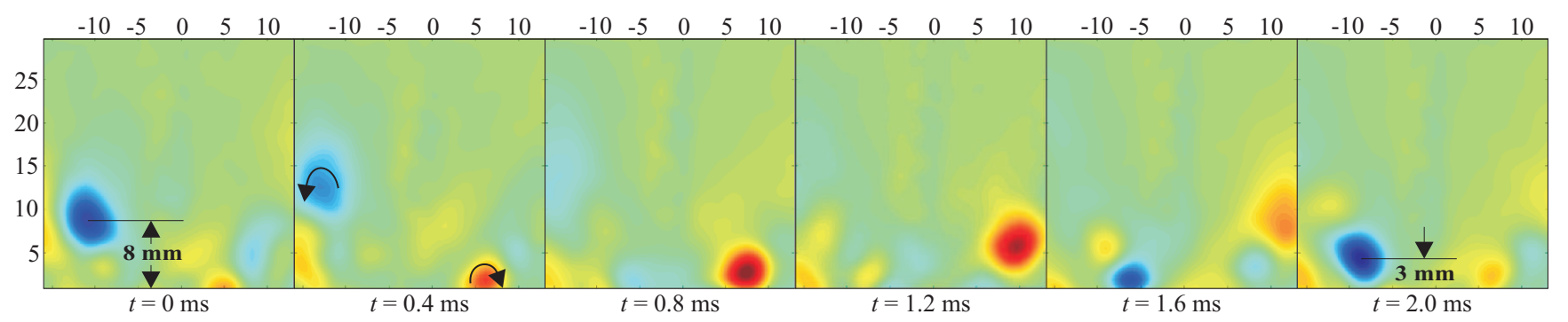

Figure 3: Dynamics of the precessing vortex core reconstructed from the $0^{t h}-10^{t h}$ modes of the POD $\left(\vec{u}_{1} 0(t)\right)$. The PVC circumscribed the nozzle at $515 \mathrm{~Hz}$ and axially stretched at the thermo-acoustic frequency of $308 \mathrm{~Hz}$. The flow is visualized in terms of the resolved vorticity $\left(\omega_{z}\right)$ between $-20000 \mathrm{~s}^{-1}$ (blue) and $20000 \mathrm{~s}^{-1}$ (red). Axes units are $\mathrm{mm}$.

kinetic energy. That is, the sum of the highest $k$ eigenvalues for the POD basis is larger than for any other orthogonal basis. Assuming that the eigenvalues are sorted in descending order, the first modes represent the dominant flow features. It is common practice in the application of POD to center the data about the mean such that the first (most energetic) mode represents a fluctuation. However, the mean flow-field shape, represented by the streamlines in Fig. 1, oscillated in intensity due to the coupling of the combustion chamber with the reactant feed system. The POD therefore was performed on the complete data set in order to explicitly capture this fluctuation. The temporal history of the velocity-field from the $k$ most dominant modes is given by $u_{k}\left(x, y, t_{i}\right)=\sum_{j=0}^{k} a_{j}\left(t_{i}\right) M_{j}(x, y)$.

The $0^{\text {th }}$ POD mode was identical to the mean field shown in Fig. 1. Spectral analysis of $a_{0}(t)$ using a fast Fourier transformation (FFT) showed a single, large amplitude fluctuation at exactly the thermo-acoustic frequency, $308 \mathrm{~Hz}$. This fluctuation represented a periodic variation in flow rate into the combustion chamber due to acoustic coupling with the reactant supply and will be discussed in more detail below. The first and second modes $(j=$ 1 and 2) were anti-symmetric turbulent modes representing the PVC. The power spectra of $a_{1}(t)$ and $a_{2}(t)$ were qualitatively identical to those presented in Ref. [50] and showed strong peaks at $515 \mathrm{~Hz}$ and $207 \mathrm{~Hz}$. The former peak represented the precession of the vortex core around the burner nozzle. The latter peak was at the difference between the PVC and thermo-acoustic frequency, which will be shown to be very important.

The dynamics of the PVC as it precessed once around the nozzle are shown in Fig. 3. This time sequence was reconstructed from the $0^{\text {th }}-10^{\text {th }}$ POD modes, which contained over $90 \%$ of the kinetic energy occurring at a coherent frequency and over $70 \%$ of the total kinetic energy of the flow. Hence, these images represent the dynamics of the PVC, with the majority of the stochastic turbuence removed. The flow is visualized in terms of the out-ofplane vorticity $\left(\omega_{z}\right)$, which was calculated by integrating the circulation around each measurement point. As can be seen, the PVC precessed once around the nozzle in approximately $2 \mathrm{~ms}$, agreeing with the results of the frequency analysis. During this precession, the helical vortex under-

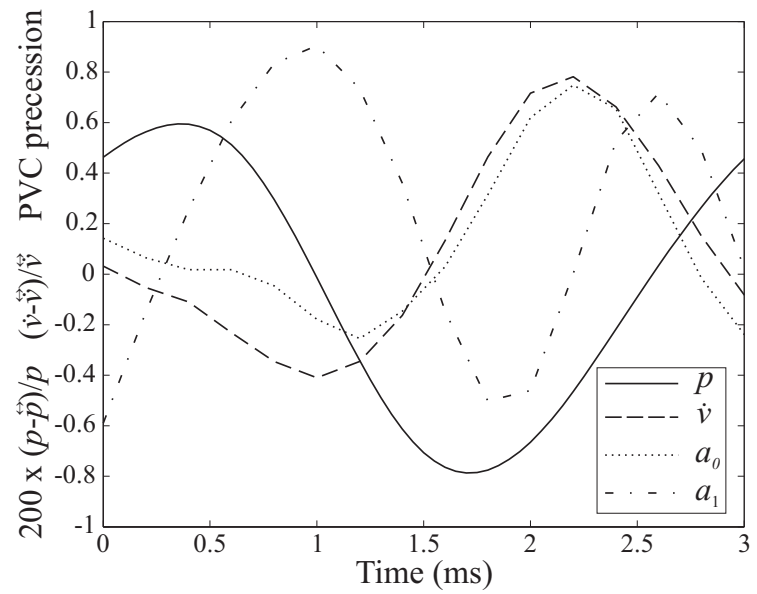

Figure 4: Temporal history of the pressure, volumetric flow rate into the combustor, and PVC precession angle during the time sequence shown in Fig. 3.

went significant contraction in the axial direction. The half-period of this contraction was approximately $1.6 \mathrm{~ms}$, corresponding to half of the thermo-acoustic cycle. This aspect of the PVC dynamics will be discussed in more detail in Section 4, where it is shown that the axial PVC dyanmics are periodic over the thermo-acoustic cycle.

Figure 4 shows a short temporal sequence of the acoustic fluctuation, the volumetric flow rate $(\dot{v})$ fluctuation, and the first two POD temporal coefficients. All fluctuations were taken relative to the long-time average (over many acoustic cycles), which is denoted $(\leftrightarrow)$. The temporal origin in this figure is the same as that for the PVC motion in Fig. 3. The volume flow rate was approximated by integrating the velocity across the nozzle exit. At some instants the inner recirculation zone penetrated into the central nozzle. Hence, only locations of positive axial velocity were included in the volume flow rate calculation to accurately represent the flow of fresh reactants into the combustion chamber. Spectral analysis showed that $\dot{v}$ fluctuated at solely the thermo-acoustic frequency, $308 \mathrm{~Hz}$. As can be seen from Fig. 4, the flow rate of reactants into the combustion chamber underwent significant fluctuations of over $50 \%$ of the mean and lagged the acoustic fluctuations 
by approximately $225^{\circ}$, which is consistent with the results of Ref. [39]. The temporal coefficient of the $0^{\text {th }}$ POD mode matched the phase and frequency of the mass flow rate fluctuation. Comparing Figs. 3 and 4, axial stretching of the PVC occurred at a time of high pressure and contraction occurred at a time of low pressure. This aspect of the oscillating flow field due to coupling of the thermoacoustic pressure fluctuations with the nozzle and reactant feed system also occurs in practical gas turbine engines [7].

It previously has been shown that the PVC frequency increases linearly with the bulk flow rate [13]. It therefore is interesting that the PVC frequency in this flame was very steady, with a full-width-at-half-maximum in the spectral peak of only a few Hertz, despite the fact that the instantaneous flow-rate into the combustion chamber could vary by over $50 \%$ during a thermo-acoustic cycle. This indicates that, while the overall precession frequency varies linearly with flow-rate, it is insensitive to high-frequency flow-rate fluctuations. There was no evidence of a widely varying PVC frequency in any mode of the POD analysis or from individual measurement sequences.

The overall flow-field dynamics of this combustor therefore can be regarded as the sum of several motions:

1. A periodic fluctuation of the reactant flow rate into the combustion chamber at the thermo-acoustic frequency of $308 \mathrm{~Hz}$

2. The precession of the helical vortex core around the burner nozzle at $515 \mathrm{~Hz}$

3. The stretching and contraction of the PVC in the axial direction at $308 \mathrm{~Hz}$

4. Stochastic turbulence overlaid on these periodic fields

\subsection{Heat release rate dynamics}

Spontaneous emission, or chemiluminescence, from $\mathrm{OH}^{*}$ occurs primarily in the reaction zone and can be taken as a qualitative indicator of the heat release rate, $\dot{q}[8]$. Spectral analysis in Ref. [50] showed that the total chemiluminescence signal in this flame, integrated over the combustor volume, fluctuated at exactly the thermo-acoustic frequency. Figure 5 shows the temporal evolution of the chemiluminescence field over a short time period, during which the signal moved around the burner. To describe this motion, the intensity-weighted chemiluminescence centroid $\left(x_{q c}, y_{q c}\right)$ was calculated for each recorded image. Figure 6 shows temporal histories of the total chemiluminescence signal, the acoustic signal, and the radial centroid location over the time period in Fig. 5. As can be seen, the total chemiluminescence intensity fluctuated at the thermo-acoustic frequency, with intensity peaks separated by $3.2 \mathrm{~ms}$. However, the centroid moved around the combustor over a time span that was longer than the thermo-acoustic frequency. Figure 7 shows the power spectrum of the radial and axial centroid locations. The location of the heat release circumscribed the combustor at exactly $207 \mathrm{~Hz}$, which is the difference between

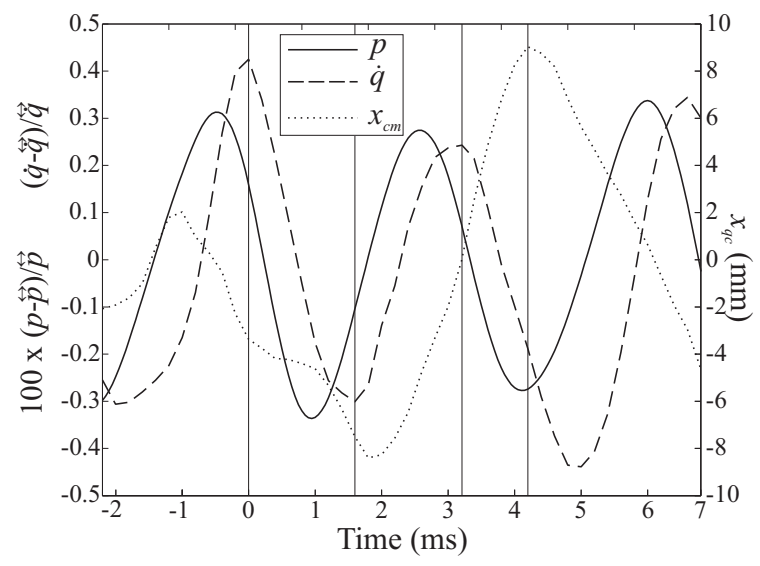

Figure 6: Temporal history of the pressure, heat release rate, and heat release rate centroid radial-position. The times shown in Fig. 5 are indicated by the vertical lines.

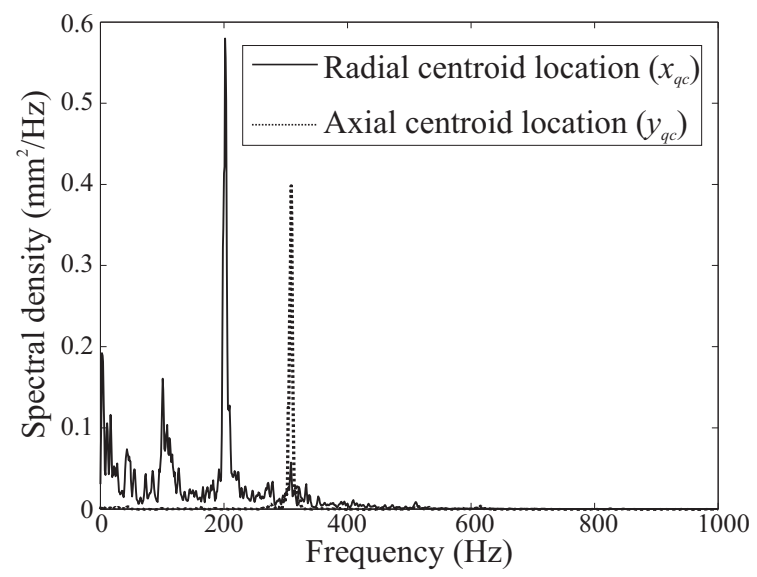

Figure 7: Power spectrum of the radial and axial locations of the intensity-weighted heat release centroid, $x_{q c}$. The radial location moved around the combustor at 207 $\mathrm{Hz}$, which is the difference between the PVC precession frequency and the thermo-acoustic frequency. The axial location fluctuated at the thermo-acoustic frequency, 308 $\mathrm{Hz}$.

the thermo-acoustic frequency and the PVC precession frequency. The heat release location also moved axially at the thermo-acoustic frequency.

The frequency of the radial motion shows that the location of the heat release fluctuation was greatly affected by the PVC. That is, as the PVC axially contracted and extended over the thermo-acoustic cycle, it caused a periodic asymmetry in the flame surface. In a PVC fixed frame, this asymmetry moved around the burner at the thermo-acoustic frequency and in the opposite direction of the PVC motion. Hence, the frequency of the heatrelease asymmetry in the laboratory frame circumscribed the combustor at the difference between the PVC and thermo-acoustic frequencies. This asymmetry will be discussed in more detail below. However it clearly can be seen 


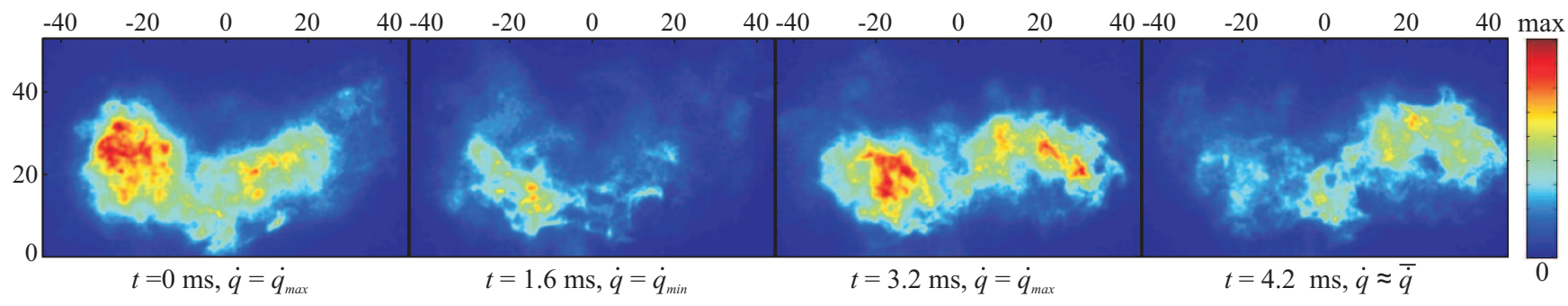

Figure 5: Typical time sequence of $\mathrm{OH}^{*}$ chemiluminescence, which represents the integrated heat release rate. Axes units are $\mathrm{mm}$.

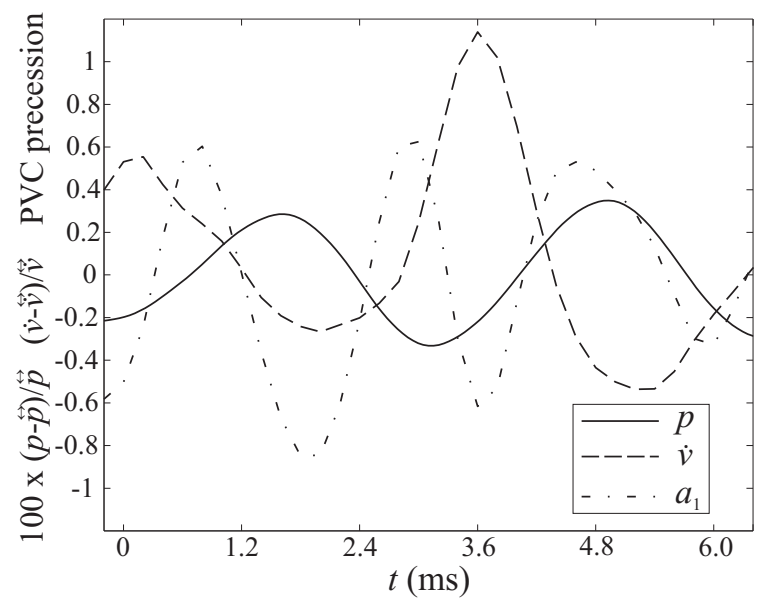

Figure 9: Typical temporal histories of the pressure, volume flow rate, and PVC precession during the same time sequence as in Fig. 8 .

that, in order to phenomenologically describe the flowflame interaction from planar measurements, the phase of the thermo-acoustic cycle and the PVC position relative to the measurement plane must be considered.

\subsection{Planar flame and flow dynamics}

Before analyzing the flow-flame interactions in detail, it is useful to qualitatively observe their dynamics from the planar high-speed measurements. Figure 8 shows a typical sequence of OH PLIF images and velocity-fields over an acoustic cycle; Figure 9 shows the temporal history of the volume flow rate into the combustor, pressure, and PVC precession over this time sequence. It must be remembered that the flow was swirling at a high rate and the image sequence does not show the evolution of a particular section of fluid with time.

The flow-field is visualized in terms of the vorticity $\left(\omega_{z}\right)$ and the axial velocity $\left(u_{y}\right)$. In each flow-field image, the thick black line represents the instantaneous reaction layer topography, which was mapped from the gradient of the $\mathrm{OH}$ signal. Determining the topography of reaction layers from $\mathrm{OH}$ PLIF images is challenging because $\mathrm{OH}$ exists not only in the reaction zone, but in high temperature products and products mixtures above approximately $1500 \mathrm{~K}[44,63]$. However, Sadanandan et al. [44] observed that $\mathrm{OH}$ exists in super-equilibrium quantities immediately downstream of the flame surface, as is found in a laminar premixed flame. The $\mathrm{OH}$ signal gradient associated with the reaction zone was much higher than the gradient in the burnt gases associated with the decay toward equilibrium. Hence, they observed that the reaction layers can be identified by regions of high $\mathrm{OH}$ gradient. An algorithm therefore was developed for this work that robustly detects the flame surface topography and converts it into mathematically treatable entities using the procedure outlined in Appendix B. It is noted that the flames thus determined are not a direct measure of the reaction rate. The reaction rate may vary along the flame due to local stratification (i.e. non-homogeneous equivalence ratio), strain-rate, and preheating due to product-gas recirculation. Nevertheless, the derived topography is sufficient to study many mechanisms of thermo-acoustic instability. Mathematically, each reaction layer was treated as a parametrically defined contour, $f(\varphi)=x_{f}(\varphi) \hat{e}_{x}+y_{f}(\varphi) \hat{e}_{y}$, where $\hat{e}_{x}$ and $\hat{e}_{y}$ are the respective unit vectors in the radial and axial directions.

The $\mathrm{OH}$ and velocity-field images in Fig. 8 exhibit many important characteristics. The most intense $\mathrm{OH}$ signal was near the nozzle exit, where hot products from the inner recirculation zone mixed rapidly with fresh gas to stabilize strong combustion. At the beginning of the sequence, the fresh reactants, indicated by the region of low $\mathrm{OH}$, did not penetrate deeply into the combustion chamber and the flame was anchored near the burner nozzle. The branch of the PVC on the left of the combustor was near the flame-front and radially deflected the incoming reactants but did not cause local flame surface wrap-up. This time corresponded to a surge of reactants into the combustor, i.e. a high volumetric flow rate as indicated by Fig. 9. After a time lag, this surge caused the swirling reactant flow to penetrate deeper into the chamber and caused increased corrugation in the flame surface. A distinct asymmetry in the flow- and $\mathrm{OH}$-fields occurred, with the reactant flow on the left side oriented more horizontally with respect to the combustor base. The reactant flow on this side of the combustor penetrated closer to the wall and combustion occurred along the bottom of the reactant jet (in the outer recirculation zone). At $t=2.4 \mathrm{~ms}$, a flame segment that had been wrapped up by the PVC convected into the plane. The highly swirling flow pro- 

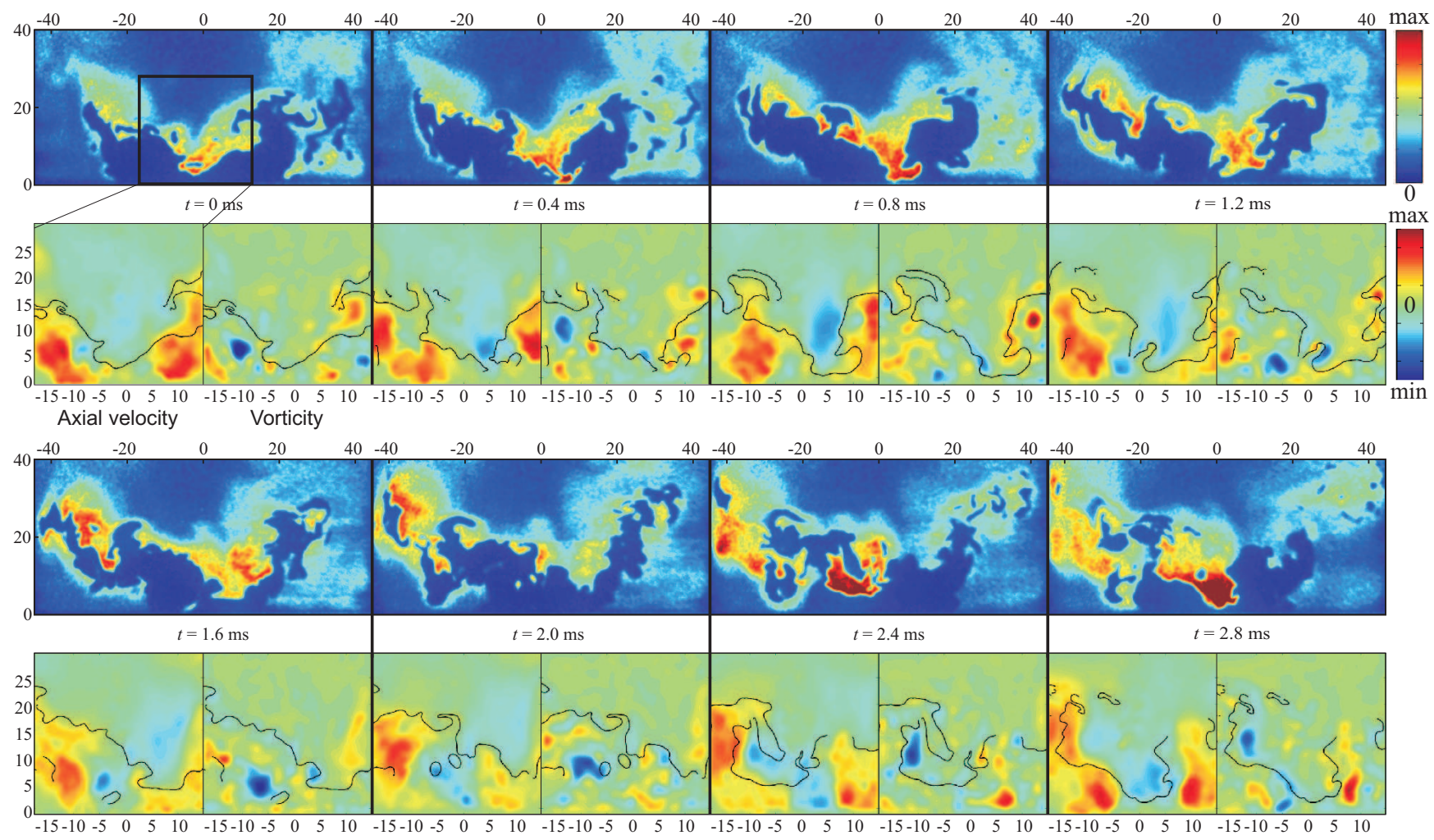

Figure 8: Typical time sequence of $\mathrm{OH}$ distribution (top images) and velocity-field (bottom two images). The background color for velocity-field images on the left is the axial velocity between -30 and $30 \mathrm{~m} / \mathrm{s}$ and the background color for the images on the right is the out-of-plane vorticity between -20000 and $20000 \mathrm{~s}^{-1}$. The thick black line represents the instantaneous flame surface topography. Axes units are mm.

gressed around the combustion chamber by approximately $220^{\circ}$ during this time sequence, estimated from the tangential velocity. Hence, the apparent asymmetry on the left of the combustor was distributed in space and swirled around the combustion chamber. To better understand this asymmetry and the combustor dynamics in general, the relative phase of the PVC with respect to the acoustic signal and measurement plane must be considered.

\section{Doubly phase resolved analysis}

Since the interaction of the PVC and the flame over an acoustic cycle has a large effect on the heat release rate, correctly interpreting the interaction from planar measurements requires simultaneous consideration of both the acoustic fluctuation and the position of the PVC with respect to the measurement plane. This can be achieved by resolving the temporal measurement sequence with respect to the phase of both phenomena. The phase in the acoustic oscillation $\left(\phi_{a}\right)$ was determined from the microphone measurements. Over each acoustic cycle, the time at which the pressure fluctuation crossed zero from positive to negative was taken as the beginning of the cycle. The period of the cycle was then divided into eight discreet phase angles. The same process was followed to determine the phase of the PVC with respect to the measurement plane $\left(\phi_{p}\right)$. The PVC oscillation was measured from the temporal coefficient of the first POD mode, $a_{1}(t)$. This coefficient was sinusoidal, with a frequency of $515 \mathrm{~Hz}$, as was shown in Figs. 4 and 9. Each temporally resolved measurement then was assigned to its closest discrete phase angle in both the acoustic and PVC cycle. This resulted in 64 different acoustic/PVC phase angle combinations describing the repeatable motion in the combustor. The locations of the assigned phase angles over a cycle are shown in Fig. 10. It is noted that the PVC phase angle is a measure of the position of the PVC from an arbitrary starting point relative to the measurement plane. Choice of this starting point does not affect the results as long as a consistent definition is used. For this work, the time when $a_{1}(t)$ crossed zero from positive to negative was taken as the zero phase angle for the PVC motion.

Huang et al. [29] provided a mathematical framework for the study of swirl flame dynamics by decomposing each variable into three parts: a long time average, a periodic fluctuation at the thermo-acoustic frequency, and a random turbulent fluctuation. Here, a similar decomposition is performed that also accounts for the phase of the PVC relative to the measurement plane. Any measured variable, $\zeta$, can be decomposed into three parts, a long time average $\overleftrightarrow{\zeta}$, a doubly phase resolved fluctuation $\zeta^{a p}\left(\phi_{a}, \phi_{p}\right)$, 


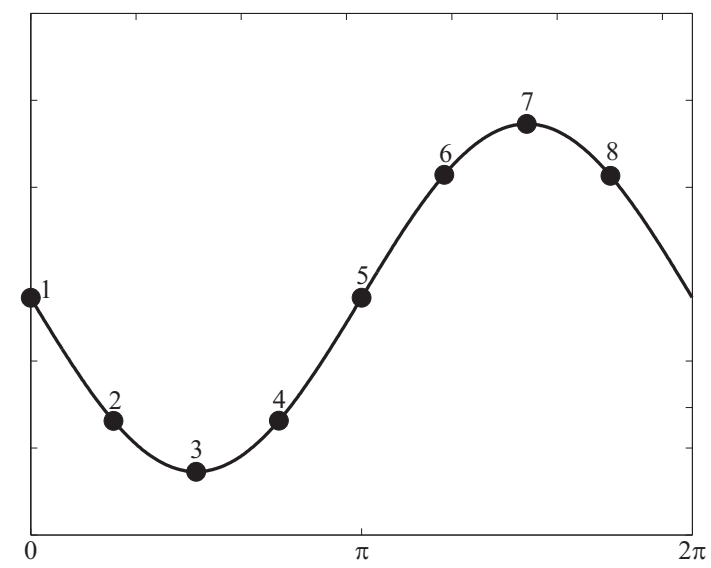

Figure 10: Definition of phase angles for periodic analysis.

and a turbulent fluctuation $\zeta^{t}(t)$, such that

$$
\zeta(t)=\overleftrightarrow{\zeta}+\zeta^{a p}\left(\phi_{a}, \phi_{p}\right)+\zeta^{t}(t)
$$

With the phase angles stipulated in Fig. 10, the decomposition produces 64 phase angle combinations and hence 64 values of $\zeta^{a p}\left(\phi_{a}, \phi_{p}\right)$. For notational convenience, the sum of the long time average and doubly phase resolved component will be denoted as

$$
\overleftrightarrow{\zeta}^{a p}\left(\phi_{a}, \phi_{p}\right)=\overleftrightarrow{\zeta}+\zeta^{a p}\left(\phi_{a}, \phi_{p}\right)
$$

the total fluctuation relative to the long time average will be denoted as

$$
\zeta^{a p t}\left(\phi_{a}, \phi_{p}, t\right)=\zeta^{a p}\left(\phi_{a}, \phi_{p}\right)+\zeta^{t}(t)=\zeta-\overleftrightarrow{\zeta}
$$

and a periodic fluctuation resolved only with respect to the acoustic phase angle will be denoted as

$$
\zeta^{a}\left(\phi_{a}\right)=\frac{1}{2 \pi} \int_{0}^{2 \pi} \zeta^{a p}\left(\phi_{a}, \phi_{p}\right) d \phi_{p}
$$

\subsection{Doubly phase resolved flow-field and combustion statis- tics}

Figure 11 shows the doubly phase resolved mean vorticity$\left(\overleftrightarrow{\omega}_{z}^{a p}\right)$ and velocity-fields $(\stackrel{\leftrightarrow}{\vec{u}})$ at one particular phase of the PVC cycle $\left(\phi_{p}=1\right)$ and at three different phases of the acoustic cycle corresponding to the pressure maximum $\left(\phi_{a}=7\right)$, mean $\left(\phi_{a}=1\right)$, and minimum $\left(\phi_{a}=3\right)$. Also shown is a black line representing the doubly phase resolved reaction layer position. This was calculated by dividing the radial coordinate into $2 \mathrm{~mm}$ bands and determining the mean axial position of the reaction layers in each band. While this contour does give a general idea of the flame shape, it does not account for regions where the flame statistically had multiple simultaneous axial locations due to wrap-up or auto-ignition pockets. Such cases

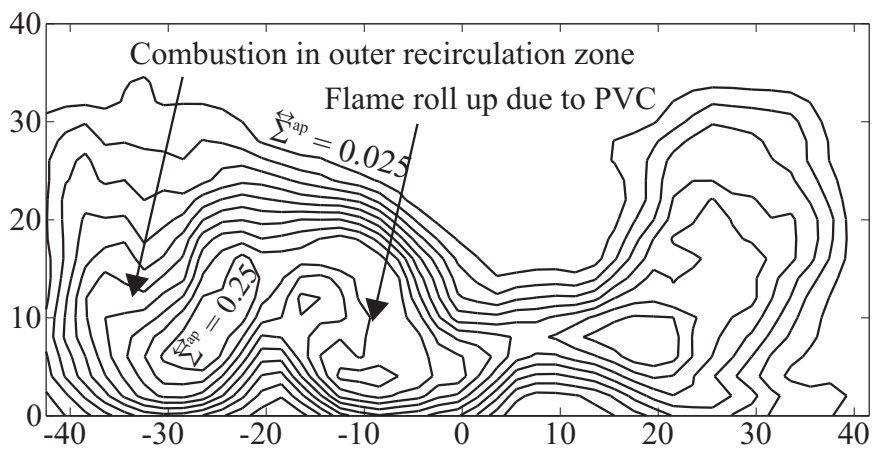

Figure 12: Doubly phase resolved flame surface density field at the same PVC and acoustic angles as Fig. 11(b) $\left(p^{a}=0,\left(\phi_{a}, \phi_{p}\right)=(1,1)\right)$. Axes units are mm.

will be elucidated below by simultaneously considering the doubly phase resolved mean flame surface density, $\stackrel{\leftrightarrow}{\Sigma}$. The standard deviation relative the doubly phase resolved velocity fields at every measurement point was typically around $50 \%$ of the mean. Approximately 125 measurements were available at each phase angle combination. Hence, the uncertainty in the mean fields is expected to be less than about $5 \%$ (i.e. $0.5 / 125^{1 / 2}$ ). The statistical accuracy of the doubly phase resolved mean fields for representing the major flow features is further demonstrated in Section 4.2.

Several important observations can be made from the flow-fields in Fig. 11. As in Fig. 3, the axial extension and contraction of the PVC over the acoustic cycle is clearly shown, with the axial distance between the PVC branches varying between approximately $13 \mathrm{~mm}$ and 6 mm. Likewise, the PVC was axially extended at the maximum chamber pressure and contracted at the minimum chamber pressure. On the left side of the combustor, the upper branch of the PVC strongly deflected the incoming flow in the radial direction, with increased deflection as the PVC contracted. This is similar to the flow-field observed at $t=0 \mathrm{~ms}$ in Fig. 8 . The PVC was close to the flame and the flow appears to be through the mean reaction location. Conversely, on the right side of the flow, the PVC was closer to the nozzle centerline and at a lower axial location. The incoming flow on this side was not deflected radially, but entered the chamber predominantly in the axial direction. The PVC on the right side of the combustion chamber was displaced from the flame and a clear stagnation line is apparent between the incoming reactants and the inner recirculation zone. The flame stabilized close to this stagnation line and accurately followed its shape.

The flame surface density can be used to further elucidate the PVC/flame interaction on the left side of the burner. The flame surface density describes the area of the reaction layers $\left(A_{f}\right)$ in a given volume $(\delta V)$ and is given by $\Sigma=\delta A_{f} / \delta V$. Here, the two-dimensional flame surface density field was calculated by dividing the measurement domain into $2 \mathrm{~mm} \times 2 \mathrm{~mm}$ cells and determining the av- 


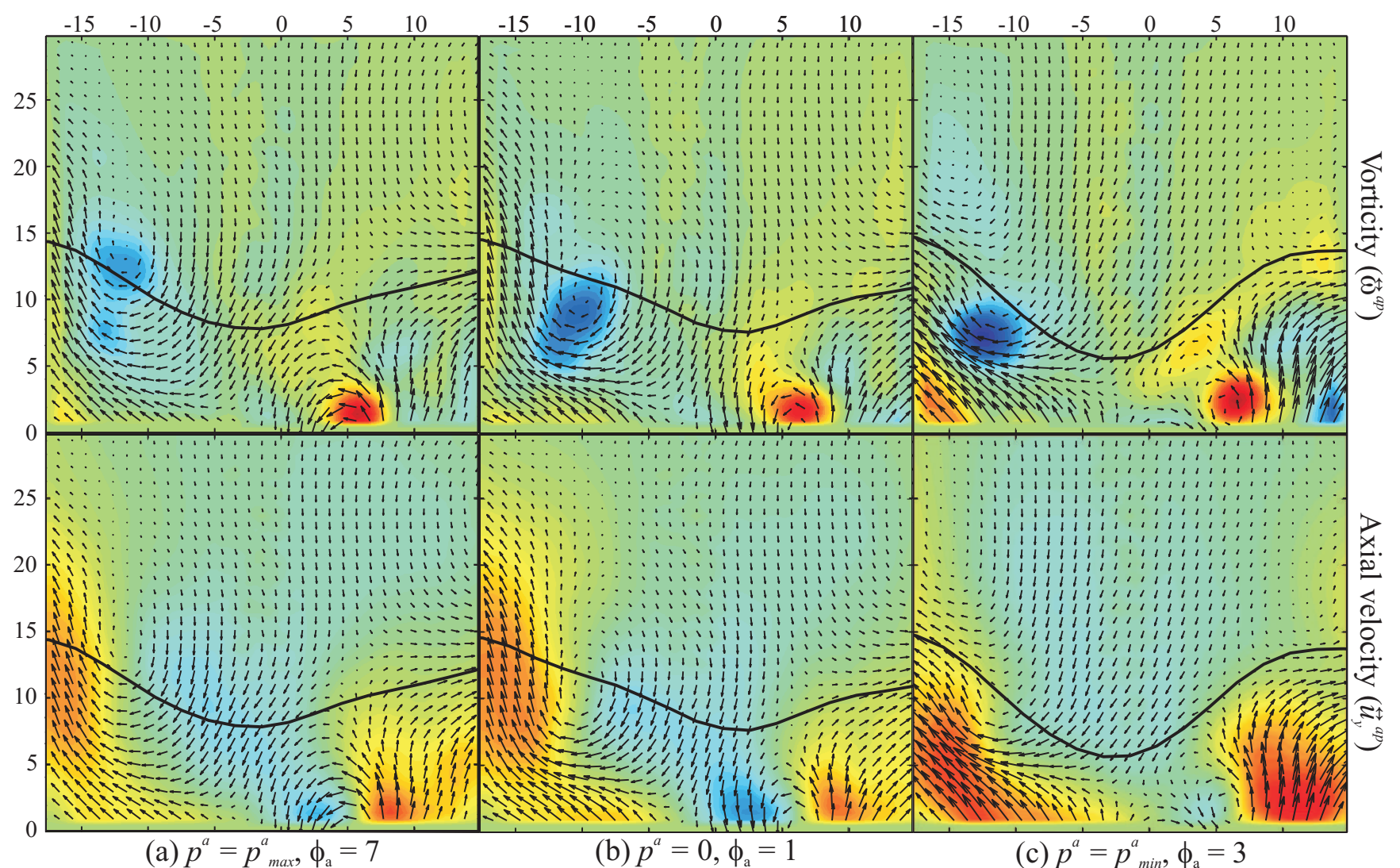

Figure 11: Doubly phase resolved flow-fields and mean flame surface locations at three phase angles of the acoustic cycle $\left(\phi_{a}=\{7,1,3\}\right)$ and one phase of the PVC cycle $\left(\phi_{p}=1\right)$. Background color in the top sequence is the vorticity $\omega_{z}$ between $-20000 \mathrm{~s}^{1}$ (blue) and $20000 \mathrm{~s}^{1}$ (red) and in the bottom sequence is the axial velocity between $-15 \mathrm{~m} / \mathrm{s}$ (blue) and $15 \mathrm{~m} / \mathrm{s}$ (red). Axes units are mm. Color map is the same as Fig. 8.

erage reaction layer length in each cell. Figure 12 shows the doubly phase resolved flame surface density, $\stackrel{\leftrightarrow}{\Sigma}^{a p}$, at $\left(\phi_{a}, \phi_{p}\right)=(1,1)$ (same as Fig. 11(b)). There was considerable roll-up of the flame surface in the region of PVC interaction, on the left of the burner. Also, there was significant combustion in the lower left corner of the burner. This corresponds to the burning around the bottom of the reactant jet (in the outer recirculation zone) that was observed in Fig. 8. Similarly, the asymmetry in the reactant inflow in Fig. 8 was due to the axial deflection of the incoming reactants on the left side of the burner caused by the PVC. This deflection and its effect on the heat release fluctuations will be discussed in more detail in Section 5 .

\subsection{Repeatability of doubly phase resolved instantaneous fields}

The distinct patterns shown in Figs. 11 and 12 indicate that there are very repeatable motions to the flow and flame when both the acoustic and PVC cycles are considered. To demonstrate this, Fig. 13 shows short time sequences of instantaneous $\mathrm{OH}$ and velocity measurements at times corresponding to the phase angles of the mean fields shown in Figs. 11(b) and 12. The time $t=0 \mathrm{~ms}$ in each image was taken at the time that corresponded exactly to the correct combination of phase angles (i.e. $\left.\left(\phi_{a}, \phi_{p}\right)=(1,1)\right)$. As can be seen, both flow and $\mathrm{OH}$ fields exhibited similar behaviors: the incoming reactants on the left were deflected radially and penetrated closer to the combustion chamber walls; there was significant combustion around the bottom of the reactant jet on the left side; the reaction layer near the nozzle exit on the left was significantly wrinkled by the PVC and that on the right was aligned along the stagnation line between the incoming flow and the inner recirculation zone.

The repeatability of this configuration is further demonstrated in Fig. 14, which shows the flame surface density field and a typical $\mathrm{OH}$ sequence taken $180^{\circ}$ further through the PVC cycle (i.e. when the PVC was in the mirrored configuration), but at the same acoustic phase angle $\left(\left(\phi_{a}, \phi_{p}\right)=(1,5)\right)$. Once again, flame surface roll up and burning in the outer recirculation zone occurred, only in this case they were on the opposite side of the combustion chamber. This figure also demonstrates the necessity of considering both the PVC and acoustic phases simultaneously. If only the acoustic phase were considered, repeatable configurations such as this would not appear and the statistics would not accurately represent the instantaneous flow-fields and flame. 

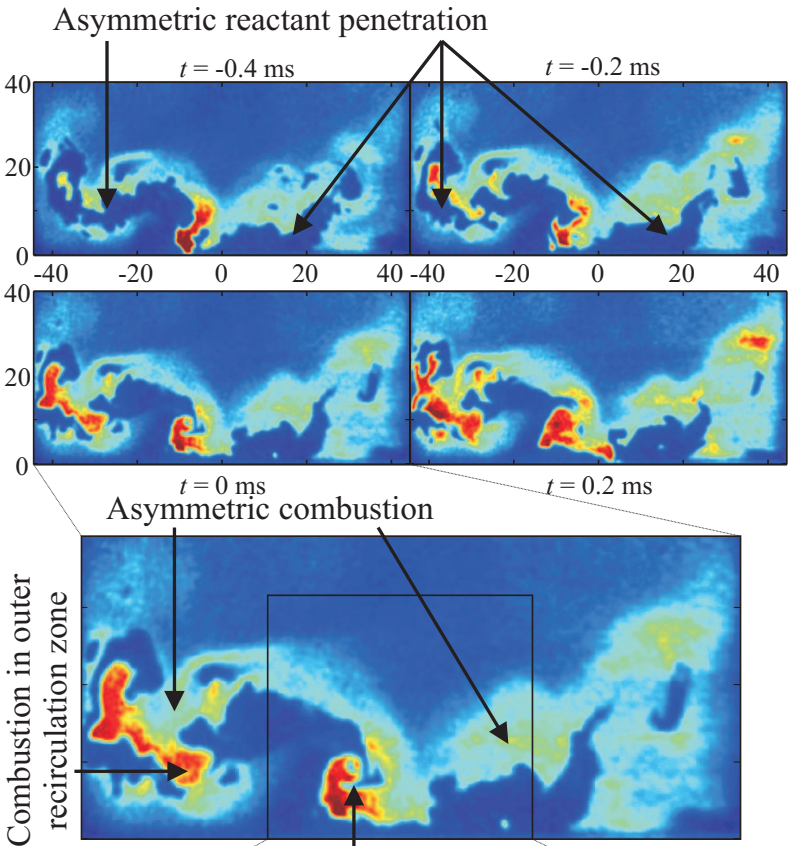

Flame surface

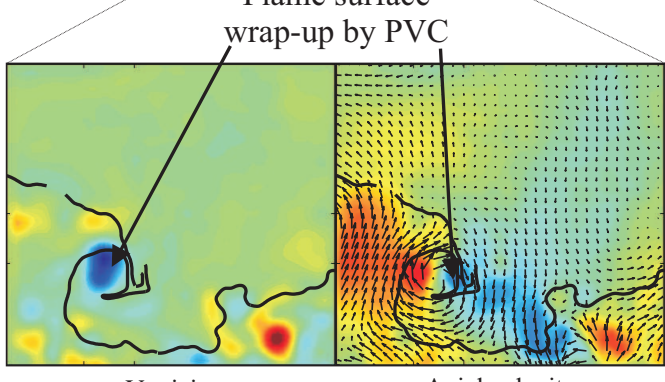

Vorticity

Axial velocity
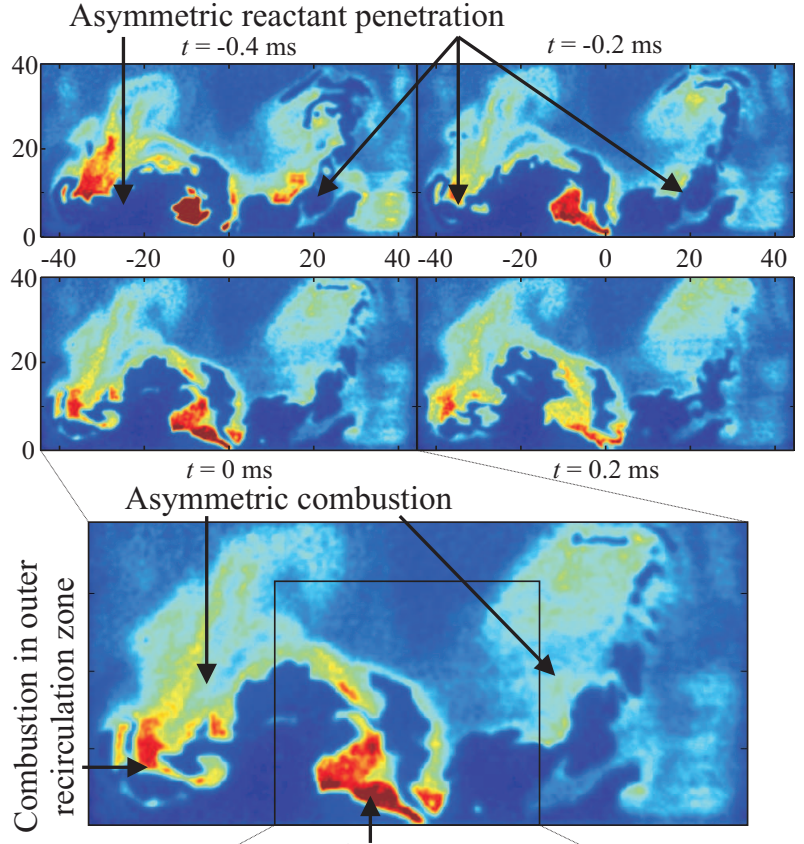

Flame surface

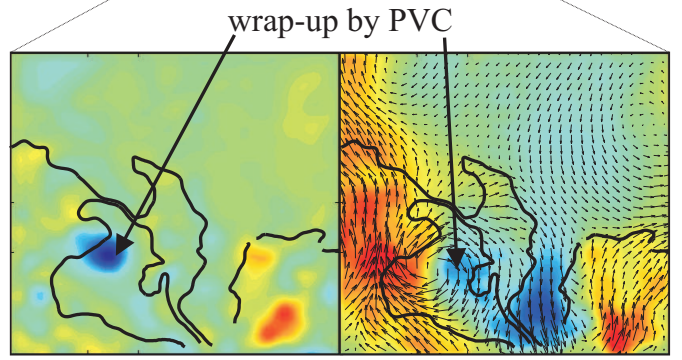

Vorticity

Axial velocity

Figure 13: Time sequences of typical $\mathrm{OH}$ fields and velocity measurements at times corresponding the zero pressure fluctuation phase in Fig. 11(b) and the flame surface density field in Fig. $12\left(\left(\phi_{a}, \phi_{p}\right)=(1,1)\right.$ at $t=0$ ms $)$. Axes units are $\mathrm{mm}$. Color map is the same as Fig. 8.

The axial contraction and extension of the PVC shown in Fig. 11 indicates that there is a difference in the PVC interaction with the flame over the acoustic cycle. Figure 15 shows a typical flow-field, flame, and the flame surface density distribution at the same PVC angle as previous results (Fig. 13), but at the minimum of the pressure fluctuation $\left(\phi_{a}=3\right)$. As can be seen, there was considerably less large-scale wrap-up around the PVC, less radial penetration of the reactant jet, and less combustion in the outer recirculation zone than at $\phi_{a}=1$. The varying interaction of the PVC with the flame as it contracted and stretched over the acoustic cycle therefore may contribute to the acoustically coupled heat release fluctuations. This is discussed further in Section 5 .

Since the global flow-field structures are very repeatable, a large fraction of the instantaneous measurements can be well described by the doubly phase resolved statistics. To demonstrate this, Fig. 16 shows the power spectra of different components of the velocity fluctuations:

$$
\kappa^{\xi}=0.5 u_{i}^{\xi} u_{i}^{\xi}
$$

integrated over the field-of-view. The notation $\kappa^{\xi}$ indicates the energy associated with a velocity fluctuation taken relative to one of the decomposition levels stipulated in Eq. 1. It is noted that $\kappa$ does not account for the variable density field and therefore is not truly the kinetic energy.

Figure 16a shows the power spectrum of the complete velocity fluctuations taken relative to the long time average, $\vec{u}^{a p t}$. The total velocity in the combustor fluctuated at exactly the thermo-acoustic frequency, $308 \mathrm{~Hz}$, as the reactants periodically surged into the combustion chamber. Figure $16 \mathrm{~b}$ shows the velocity fluctuations relative to the doubly phase resolved mean velocity-fields, $\vec{u}^{t}$. From this figure, it clearly can be seen that the majority of the velocity fluctuations were contained in the doubly phase resolved mean fields. There remained only a relatively low amplitude peak at $308 \mathrm{~Hz}$, which represented the varying stochastic turbulence (cycle to cycle randomness) between acoustic periods. That is, the oscillating reactant flow caused an oscillation in the intensity of the stochastic turbulence that still was apparent in the spectrum. How- 
Asymmetric reactant penetration
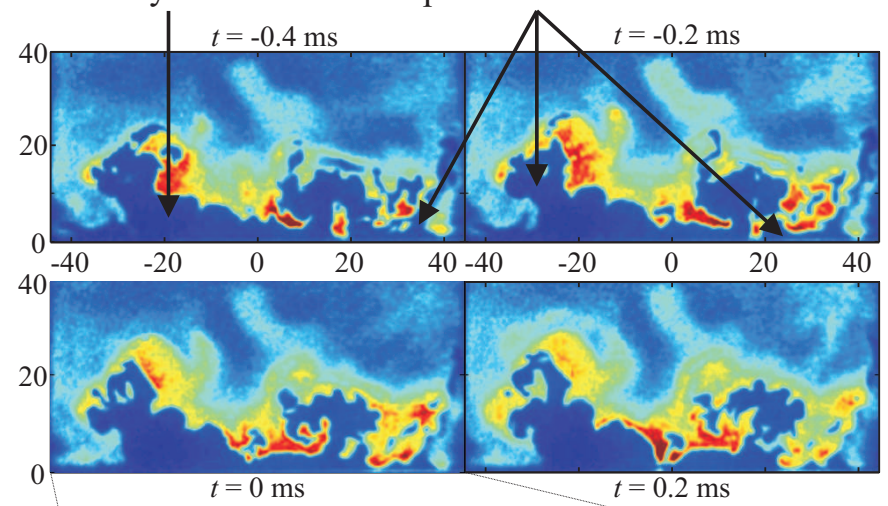

Asymmetric combustion

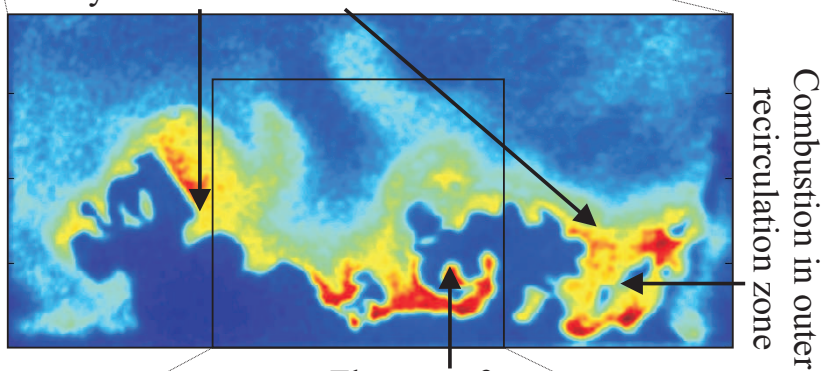

Flame surface

wrap-up by PVC

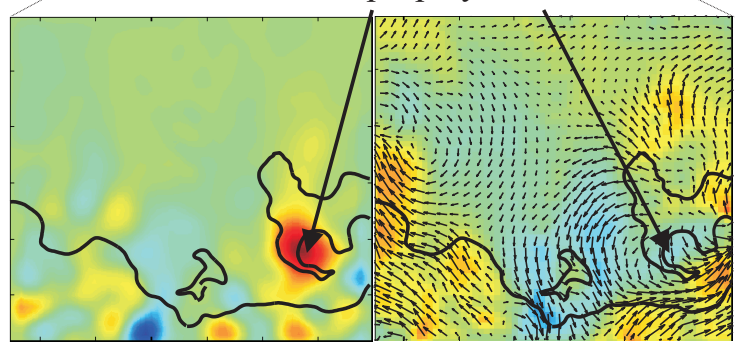

(a) Typical sequence of $\mathrm{OH}$ images, $\left(\phi_{a}, \phi_{p}\right)=(1,5)$ at $t=0 \mathrm{~ms}$

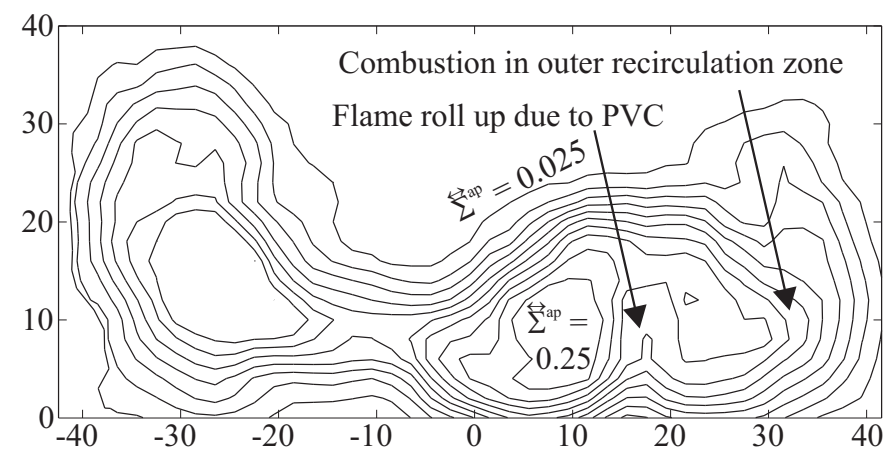

(b) Flame surface density, $\left(\phi_{a}, \phi_{p}\right)=(1,5)$

Figure 14: Doubly phase resolved measurements taken at the same acoustic phase as Fig. 13, but $180^{\circ}$ further through the PVC phase. Axes units are mm. Color map is the same as Fig. 8.

ever, the doubly phase resolved mean velocity-fields represented virtually all of the coherently oscillating velocity fluctuations in this combustor.
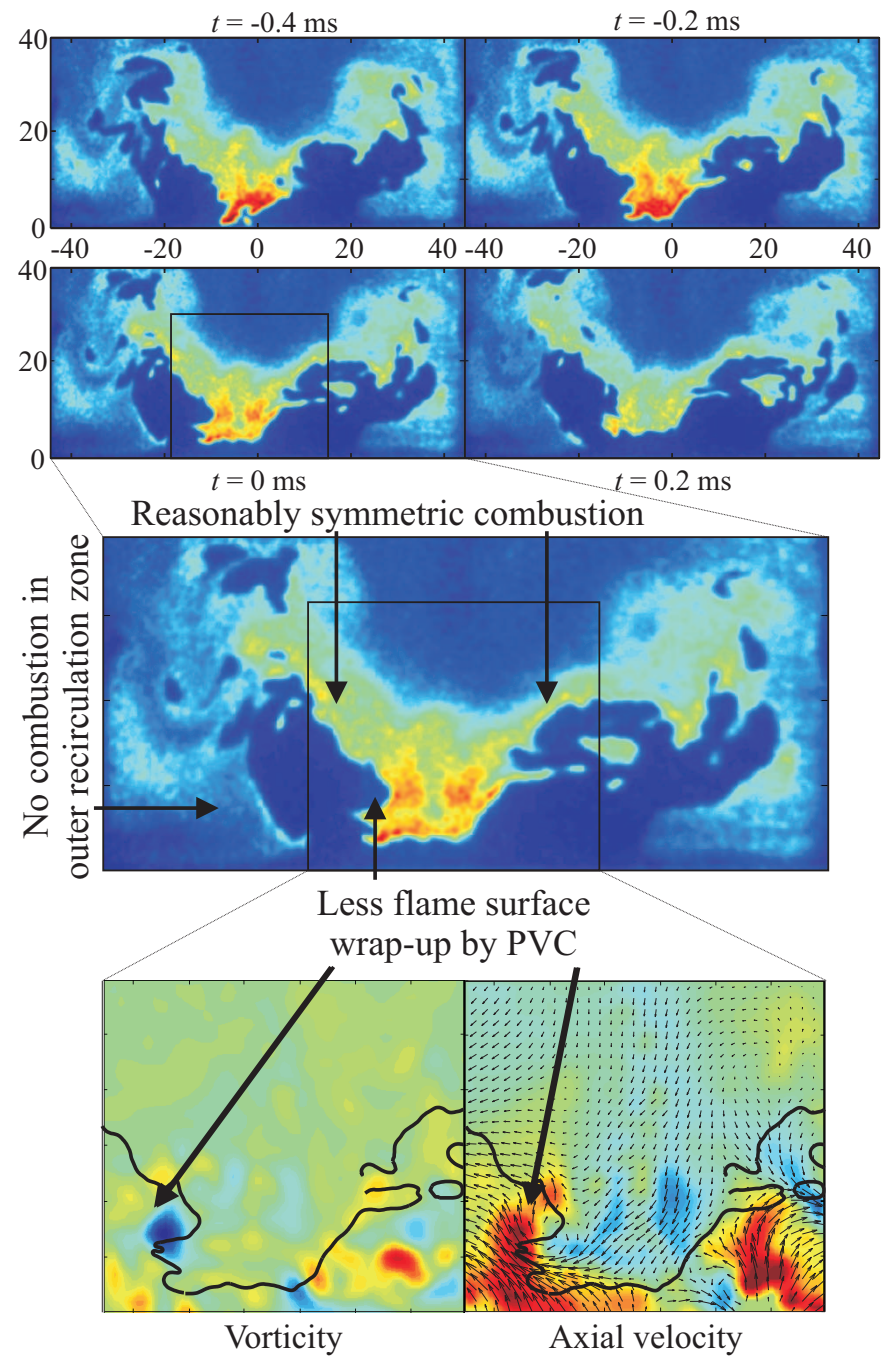

(a) Typical $\mathrm{OH}$ and flow-fields, $\left(\phi_{a}, \phi_{p}\right)=(3,1)$ at $t=0 \mathrm{~ms}$

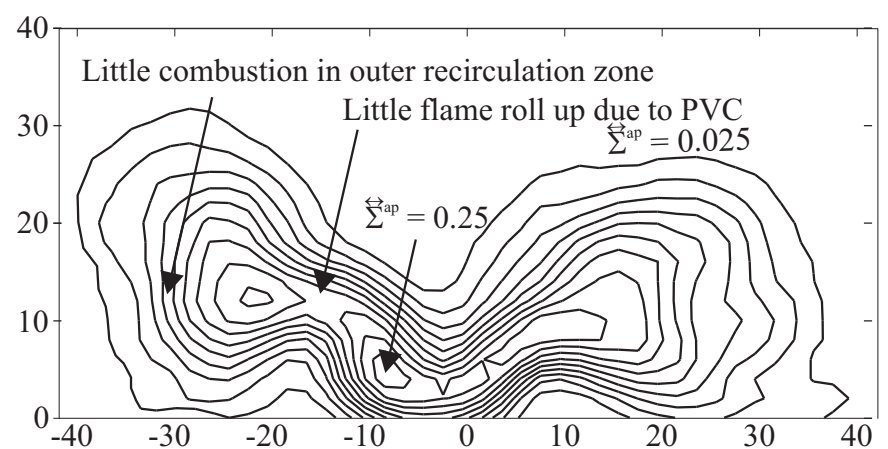

(b) Flame surface density, $\left(\phi_{a}, \phi_{p}\right)=(3,1)$

Figure 15: Doubly phase resolved results at the PVC and acoustic phases shown in Fig. 11(c). Axes units are mm. Color map is the same as Fig. 8.

\subsection{Flame orientation}

Statistical repeatability of the doubly resolved flame surface position was demonstrated in Figs. 12 and 14b in terms of the flame surface density. This can be further shown by considering the statistics of the reaction layer ori- 


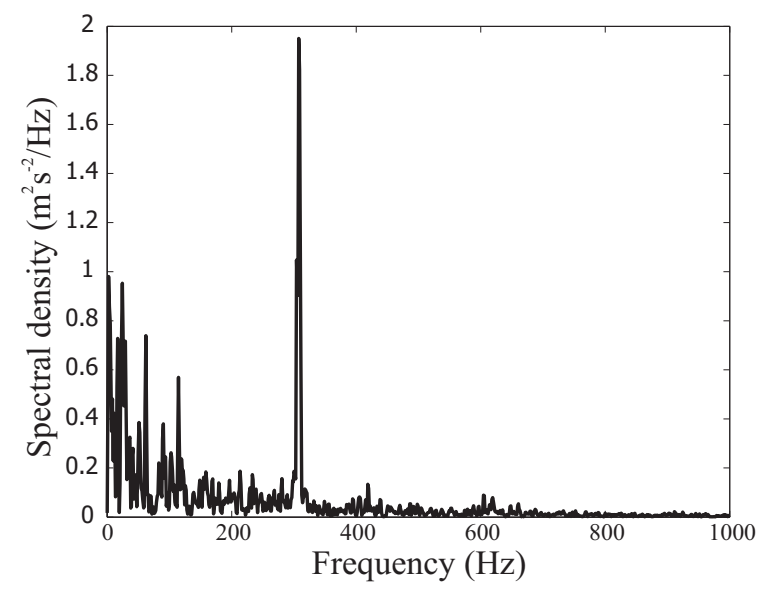

(a) Velocity fluctuation relative to long average, $\kappa^{\text {apt }}$

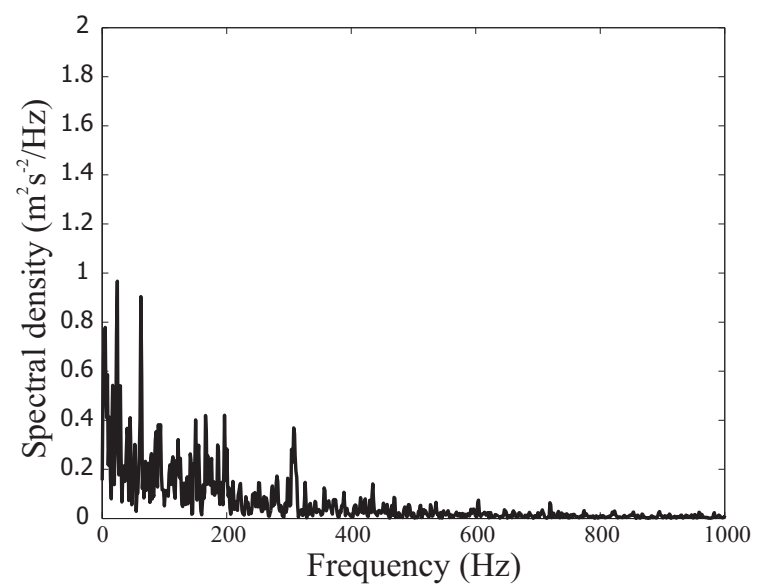

(b) Velocity fluctuation relative to doubly phase resolved mean fields, $\kappa^{t}$

Figure 16: Velocity fluctuation power spectra with the fluctuations taken relative to different periodic repeatable motions.

entation with respect to the flow-field. This relationship is important as it helps describe the location and shape of the reaction layers, which influences the phase between combustion and fluid mechanical processes. It was seen in Fig. 11 that when the PVC is not interacting with the flame, the flame tended to exist around the stagnation line between the incoming flow and the inner recirculation zone. When the PVC was interacting such as at $\left(\phi_{a}, \phi_{p}\right)=(1,1)$, Fig. 13 shows that the flame was wrapped up around the PVC. To statistically describe these different configurations, the orientation of the reaction layers with respect to the flow-field was considered. The flame surface normal vector, $\hat{n}$, was computed at every location along the instantaneous reaction layers. The inverse cosine of the inner product magnitude,

$$
\Omega=\operatorname{acos}\left(\frac{\left|\vec{u}_{f} \cdot \hat{n}\right|}{\left|\vec{u}_{f}\right|}\right)
$$

describes the orientation of flame with respect to the flow, where $\vec{u}_{f}$ is the velocity-field interpolated to the flame sur-

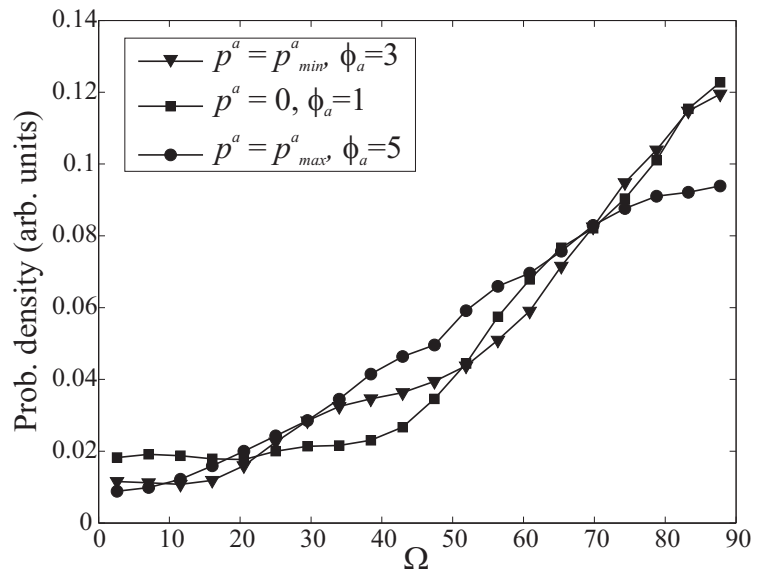

(a) $x=7.5$ to $12.5 \mathrm{~mm}$

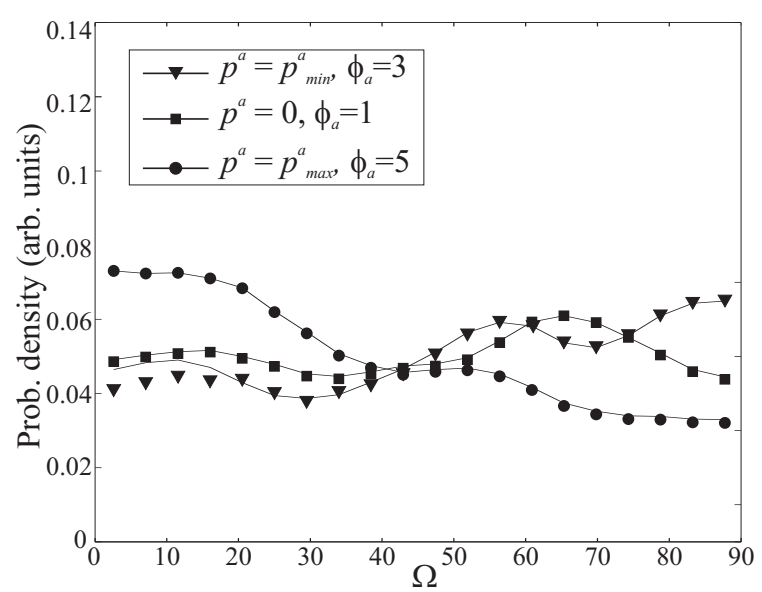

(b) $x=-7.5$ to $-12.5 \mathrm{~mm}$

Figure 17: Probability distribution functions of the orientation of the flame with respect to the flow at different radial locations, $\Omega$. The statistics were compiled at the PVC and acoustic phases shown in Fig. 11.

face. A value of $90^{\circ}$ indicates that the flame was oriented parallel to the flow (e.g. along a stagnation line), while a value of $0^{\circ}$ indicates that the flow was normal to the flame.

Figure 17 shows the probability distribution functions (PDF) of $\Omega$ taken at the three phase angle combinations in Fig. 11. The PDFs were compiled over two radial bands, $x= \pm(7.5$ to 12.5$) \mathrm{mm}$. The positive band was located where the mean flame appears to stabilize along the mean stagnation line between the incoming flow and the inner recirculation zone. The PDFs show that the instantaneous flame surface normals were oriented predominantly perpendicular to the flow; the instantaneous flame surfaces were predominantly along the stagnation line. Hence, where the PVC was not interacting with the flame, the flame stabilized between the incoming reactants and the recirculated hot products. The negative radial band was located where the PVC was interacting with the flame. The instantaneous images in Fig. 13 show that the flame was wrapped up by the PVC and that the velocity- 


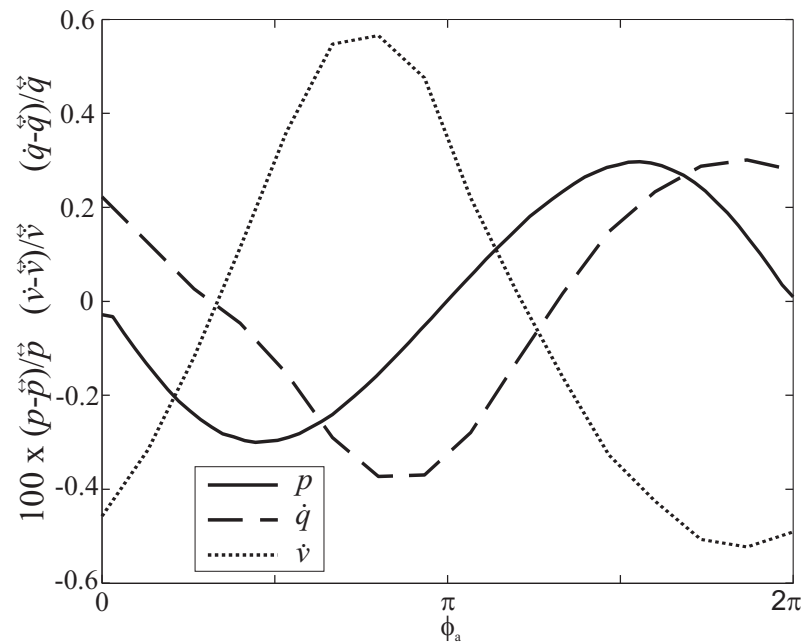

Figure 18: Acoustically phase-resolved pressure, reactant flux, and heat release fluctuations.

field was oriented both tangentially and perpendicularly to the reaction layers. The PDFs in Fig. 17b confirm this distribution of orientation. It is noted that the field-ofview for the velocity measurements was restricted to a 32 $\mathrm{mm}$ x $30 \mathrm{~mm}$ square around the nozzle exit. It therefore was not possible to determine if spectra in Fig. 16 and the PDFs in Fig. 17 are representative of the entire burner.

\section{Flow-flame interactions causing acoustically cou- pled heat release fluctuations}

The flow-flame interactions described in Sections 3 and 4 have illustrated several mechanisms that can cause thermoacoustically coupled heat release fluctuations. At the minimum combustion chamber pressure, the PVC was axially contracted and did not strongly interact with the flame in the measured field-of-view. However, Fig. 11 shows that the contracted PVC caused the largest radial deflection of the incoming reactants. As shown in Fig. 18, the minimum-pressure phase $\left(\phi_{a}=3\right)$ corresponded to a positive reactant flow-rate fluctuation. After a convective time lag, the increased reactant flux caused increased corrugation of the reaction layers due to the increased turbulence. Furthermore, the high-velocity reactant jets penetrated farther into the combustion chamber, increasing the interface between the inflow and the hot products from the inner recirculation zone. The portion of the reactant stream that was radially deflected by the PVC penetrated closer to the combustion chamber walls. Combustion occurred along the extended reactant/product interface, as well as in the outer recirculation zone below the radially-deflected stream. As the combustion chamber pressure increased, the PVC extended and interacted with the flame, causing significant flame wrinkling. It is noted that the approximately $180^{\circ}$ degree $(1.5 \mathrm{~ms})$ lag between the maximum volumetric flow rate and the maximum heat release rate seen in Fig. 18 corresponds to the time required for the

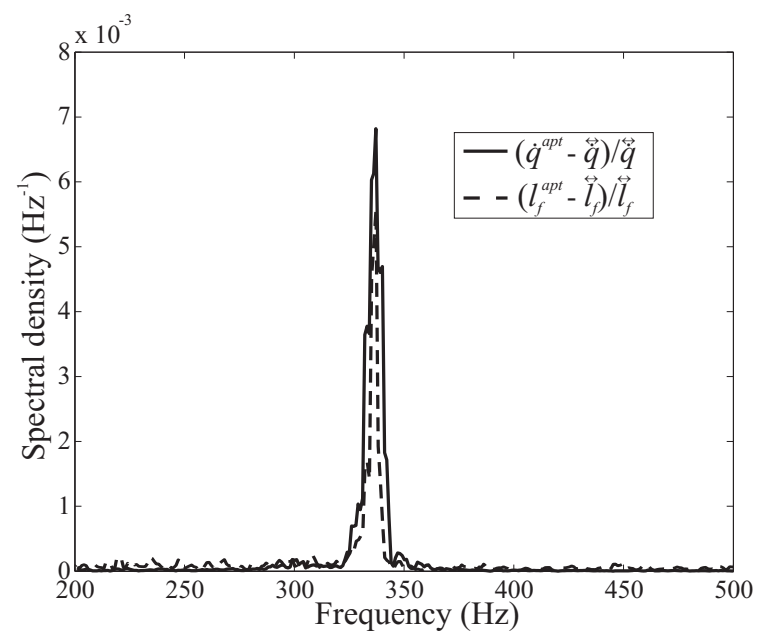

Figure 19: Power spectrum of the flame length fluctuations, $l_{f}^{a p t}$.

surging reactants to convect from the nozzle exit to the mean flame-front location $(y \approx 15 \mathrm{~mm})$ at the mean axial velocity $\left(u_{y} \approx 10 \mathrm{~m} / \mathrm{s}\right)$.

These flow-flame interactions pertain to increased heat release rate due to increased reaction layer area [29, 64, 65]. However, changes in the overall heat release rate also could be caused by changes in the reaction rate per unit area due to, for example, changes in the equivalence ratio at the flame $[66,67]$. The statistics of the reaction layer length fluctuations therefore were compared to those of the integrated $\mathrm{OH}^{*}$ chemiluminescence, which is an indicator of the heat release rate. The total reaction layer length $\left(l_{f}\right)$ was calculated from each OH PLIF image based on the topographic mapping procedure described in Appendix B. Due to the circular symmetry of the combustor, the measured planar flame length was multiplied by its radial coordinate in order to better approximate the true threedimensional flame area (i.e. $d l_{f, 3 \mathrm{D}} \sim r d l_{f \text {,planar }}$ ). The power spectrum of the flame length fluctuations, shown in Fig. 19, was then compared to that of the $\mathrm{OH}^{*}$ chemiluminescence fluctuations. For both $l_{f}$ and $\mathrm{OH}^{*}$, the fluctuation intensity in the range of $308 \mathrm{~Hz}$ was determined as the integral of the square root of the area under the power spectrum over a $2 \mathrm{~Hz}$ band $(307-309 \mathrm{~Hz})$. It was found that,

$$
\frac{l_{f}^{a p t}(308 \mathrm{~Hz})}{\overleftrightarrow{l}_{f}} \approx 0.8 \frac{\left(O H^{*}\right)^{a p t}(308 \mathrm{~Hz})}{\overleftrightarrow{\dot{q}}}
$$

implying that a significant portion of the heat release fluctuations at the thermo-acoustic frequency were caused by fluctuations in the reaction layer area. Different frequency bandwidths surrounding $308 \mathrm{~Hz}$ were checked, with no change to the results. However, the $\mathrm{OH}^{*}$ chemiluminescence fluctuations are only an approximate indicator of the total heat release fluctuations due to local stratification of this partially premixed flow. It previously has been shown that the local mixture fraction at the flame surface varies 
significantly in this combustor, regardless of the thermoacoustic phase angle [42], which effects the relationship between the measured $\mathrm{OH}^{*}$ signal and the heat release. Acoustically phase correlated measurement have shown that there is a periodic variation in the average mixture fraction of the incoming reactants [40]. These same measurements indicated that there may be a periodic change in the mean mixture fraction of the reacting fluid at the flame surface over the thermo-acoustic cycle, which would contribute to periodic oscillations in the global heat release rate by altering the local heat release rate per unit area. Such variations in the heat release rate along individual flame segments could not be measured with the present diagnostics. Nevertheless, there definitely were large fluctuations in the reaction layer length at the thermo-acoustic frequency and analysis of these fluctuations yields valuable insight into the instability mechanisms.

It therefore is necessary to analyze the mechanisms that caused changes in the reaction layer area. This was done in two parts. First, fluctuations in the total reaction layer length were considered, followed by fluctuations relative to the doubly phase resolved means.

\subsection{Fluctuations in the total reaction layer length}

In order to analyze the different mechanisms that cause changes in the flame length, one can consider their different length scales. The elongation of the reaction layers due to greater penetration of the reactant jets occurred over a length scale on the order of the combustor width. Wrinkling of the reaction layers due to interaction with the PVC occurred at a scale similar to cross-sectional diameter of the PVC branches. Finally, fine scale corrugation of the flame by stochastic turbulence happened at scales smaller than the PVC.

The relative importance of each mechanism therefore can be determined by filtering the instantaneous reaction layers at a variety of scales, removing corrugations at scales smaller than the filter size in a similar fashion to large eddy simulations. For the current data set, the flame topography was filtered using a square kernel with sizes ranging from $3 \mathrm{~mm}$ to $15 \mathrm{~mm}$. The smallest filter removed only small-scale corrugations, but resolved wrinkling at the scale of the PVC. The largest filter removed virtually all corrugations and the resultant fluctuations represented the elongation of the reaction layers. As the filter approached the endpoints of an individual flame segment, the filter size was reduced so as to not extend past the end of the flame. Flame segments that were shorter than the filter kernel were filtered using a kernel width equal to the length of the flame segment, which removed all corrugations from these segments. The largest filter size employed corresponded to approximately half the length of the longest continuous flame segments. A sample instantaneous flame surface filtered at the smallest and largest scales is shown in Fig. 20.

Power spectra of the flame length fluctuations were computed at each filter scale. The fluctuation intensity

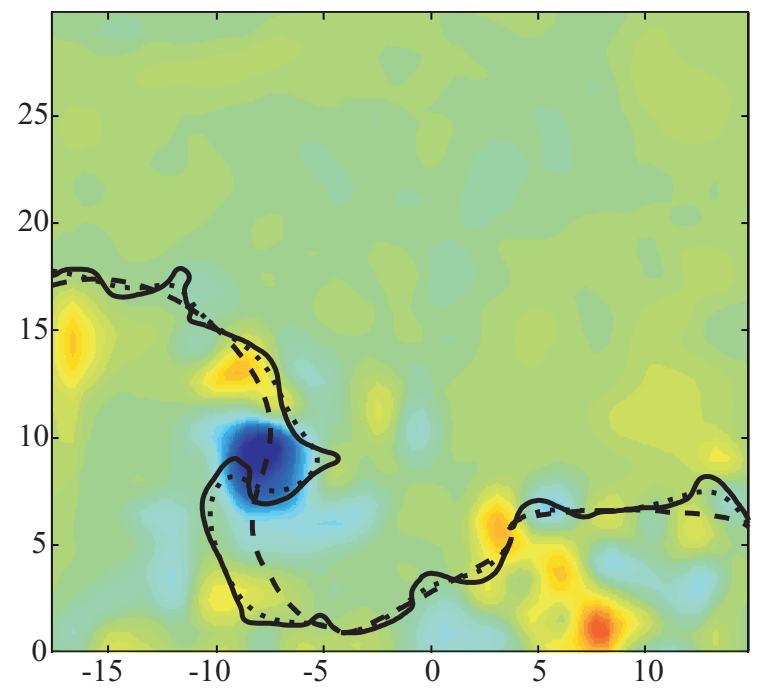

Figure 20: Sample of the measured instantaneous reaction layer topography $(-)$ and the topography filtered with a $15 \mathrm{~mm} \mathrm{(--)}$ and $3 \mathrm{~mm} \mathrm{(..)}$ square filter. Background is the vorticity field. Axes units are mm. Color map is the same as Fig. 8.

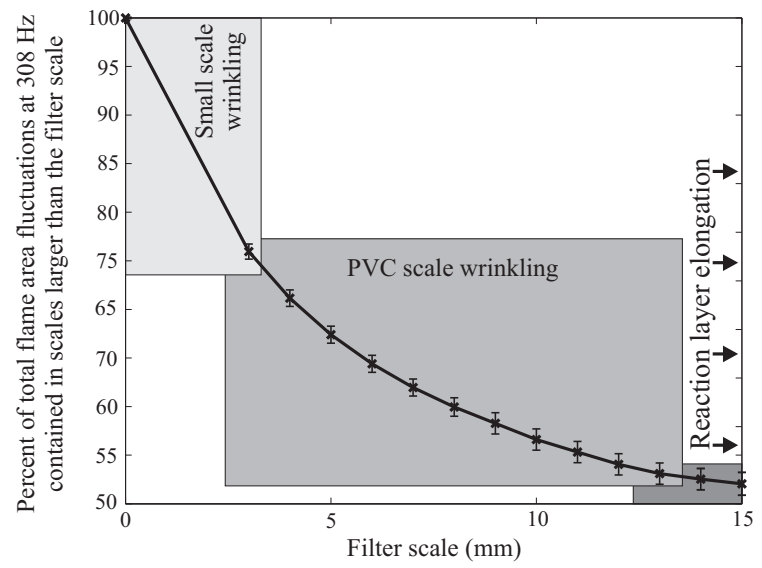

Figure 21: Distribution of flame surface length fluctuations versus filter scale. It is noted that the division into domains is not exact. The error bars represent the effect of varying the threshold level used to map the flame topography from the OH PLIF images.

in the range of $308 \mathrm{~Hz}$ was once again determined as the integrated square root of the area under each power spectrum over the range 307-309 Hz. Figure 21 shows the percentage of flame length fluctuations contained at scales larger than each filter scale. There was a sharp decrease between the unfiltered flame and the smallest filter scale, indicating that approximately $25 \%$ of the flame length fluctuations were due to small-scale, stochastic turbulence. At the largest filter scales, the curve began to asymptotically approach a value of around 50\%. These represent scales at which no wrinkling occurred and describe elongation of the flame surface due to the reactant surge. The interme- 
diate range represents large corrugations on the scale of the PVC. Of course, the exact boundaries of the different ranges are not well defined. Furthermore, the flame topography measured from the OH PLIF gradients was slightly dependent on the threshold employed. To determine this effect, the threshold was varied over a range of $\pm 10 \%$ of the value used throughout this paper. This range represents the limits for which the resultant topography visually agreed well with the apparent reaction layers. The major effect of varying the threshold was to alter the amount of flame surface in the outer recirculation zone. This changed the amount of flame length fluctuations at all scales, with the large-scale fluctuations being most affected. However, these alterations were minor and did not affect the phenomological results. The error bars in Fig. 21 represent the maximum devation caused by changing the threshold used to measure the topography. Despite any ambiguities, it clearly can be seen that the largest contributor to the flame length fluctuations was elongation of the reaction layers due to the surging flux of reactants.

\subsection{Doubly phase resolved fluctuations}

Section 4 showed that several properties of the flow and flame were highly repeatable when the phase of both the acoustics and PVC were considered. Hence, a large component of the fluctuations in flame surface length may also be highly repeatable and contained within the doubly phase resolved mean fields. To demonstrate this, the turbulent flame length fluctuation at a particular instant, $l_{f}^{t}(t)$, was calculated relative to the doubly phase resolved mean at the phase angle combination corresponding to that instant. The temporal history of $l_{f}^{t}(t)$ represents the fluctuating flame length that is not accounted for in the doubly phase resolved statistics. Figure 22 shows the power spectrum of these fluctuations. Comparing this figure to Fig. 19, it is apparent that the majority of the flame length fluctuations at $308 \mathrm{~Hz}$ were described by the doubly phase resolved statistics; the peak at $308 \mathrm{~Hz}$ decreased by approximately $90 \%$. This is consistant with the results from the velocity fluctuations shown in Fig. 16.

It also is interesting to note that the flame surface length fluctuations over the entire burner occurred at exactly the thermo-acoustic frequency. However, it was seen that the geometry of the reaction layers depended on the PVC cycle relative to the measurement plane. The absence of the PVC frequency in the flame length spectrum is because the entire combustor width was considered at once; fluctuations in the flame length were occurring somewhere in the measurement plane on every acoustic cycle. The influence of the PVC is observed when isolated regions of the combustor are considered. For example, Fig. 23 shows the power spectrum of flame length taken over the radial range $x=5 \mathrm{~mm}$ to $15 \mathrm{~mm}$. In this spectrum, the periodicity of the PVC is apparent and the flame length fluctuated at $207 \mathrm{~Hz}$. That is, flame surface variations at this location occurred when the PVC was at a particular position during the acoustic cycle, wrapping up the

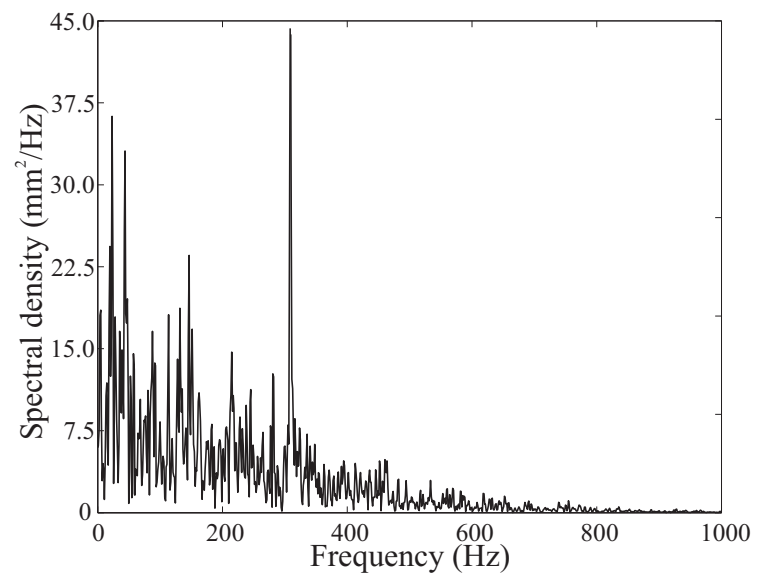

Figure 22: Power spectrum of flame length fluctuations taken relative to the doubly phase resolved means, $l_{f}^{t}$.

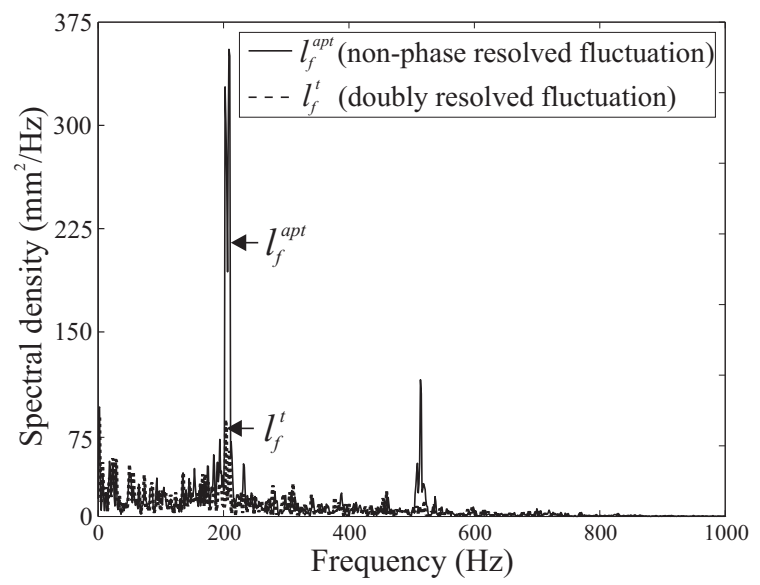

Figure 23: Power spectrum of the total flame length fluctuation and fluctuation relative to the doubly phase resolved means in the radial band $x=5 \mathrm{~mm}$ to $15 \mathrm{~mm}$.

reaction layers in the measurement plane. This figure also shows the fluctuations taken relative to the doubly phase resolved means. Once again, the doubly phase resolved measurements accounted for nearly all of the measured flame length fluctuations.

\section{Conclusions}

High-speed measurements of the three component velocityfield, planar $\mathrm{OH}$ distribution, and $\mathrm{OH}^{*}$ chemiluminescence were used to describe the flow-field, heat release, and flowflame interaction dynamics in a thermo-acoustically unstable gas turbine model combustor. The combustor was a modified version of a practical gas turbine swirl burner that was installed in an optically accessible combustion chamber. The flame, which burned a $\mathrm{CH}_{4}$ /air mixture at an equivalence ratio of 0.75 and thermal power of 10 $\mathrm{kW}$, exhibited self-excited thermo-acoustic oscillations at a frequency of $308 \mathrm{~Hz}$. 
It was found that the flow-field exhibited several distinct motions: the reactants periodically surged into the combustor at the thermo-acoustic frequency, $308 \mathrm{~Hz}$; a helical precessing vortex core circumscribed the burner nozzle at $515 \mathrm{~Hz}$; the PVC underwent axial extension and contraction at the thermo-acoustic frequency; and there was a change in the stochastic turbulence intensity at the thermo-acoustic frequency due to the surging reactants. The global heat release rate fluctuated at the thermoacoustic frequency. However, the location of the heat release centroid moved around the combustor at $207 \mathrm{~Hz}$, which is the difference between the thermo-acoustic and PVC frequencies. Hence, the interaction of the flame with the PVC had a large effect on the local heat release.

The effect of the PVC-flame interaction on the heat release motivated the compilation of doubly phase resolved statistics that jointly considered the phase of the acoustic cycle and PVC motion. These doubly phase resolved statistics revealed highly repeatable configurations and phenomena. The axial stretching of the PVC was apparent, with contraction occurring at the pressure minimum and extension at the pressure maximum. Where the PVC was not interacting with the flame, the reaction zone stabilized near the stagnation line between the incoming flow and the inner recirculation zone. At other locations, the incoming flow was deflected radially by the PVC. This caused greater penetration of the fuel jet towards the combustor walls and was associated with burning in the outer recirculation zone. At some locations and phase angles, the PVC interacted with the flame and caused significant and repeatable large-scale wrinkling.

The spectrum of the reaction layer length fluctuations showed large oscillations in the flame surface area at the thermo-accoustic frequency that significantly affected the total heat-release oscillations. By filtering the instantaneous reaction layers at different length scales, the importance of different flow-flame interactions affecting the flame length was determined. Based on the length scale, the greatest contributor appeared to be elongation of the reaction layers due to the surging reactant flow, which accounted for approximately $50 \%$ of the fluctuations. The remaining 50\% was distributed between fine scale stochastic corrugation and large-scale corrugation due to the PVC.

\section{Acknowledgments}

C. Carter acknowledges support of the Air Force Office of Scientific Research (AFOSR) Windows on Europe Program.

\section{References}

[1] S. Correa, A review of NOx formation under gas-turbine combustion conditions, Combust. Sci. Technol. 87 (1992) 329-362.

[2] S. Correa, Power generation and aeropropulsion gas turbines: From combustion science to combustion technology, Proc. Combust. Inst. 27 (1998) 1793-1807.
[3] A. Lefebvre, Gas Turbine Combustion, Taylor and Francis, 1999.

[4] S. M. Candel, Combustion dynamics and control: Progress and challenges, Proc. Combust. Inst. 29 (2002) 1-28.

[5] T. Sattelmayer, Influence of the combustor aerodynamics on combustion instabilities from equivalence ratio fluctuations, J. Eng. Gas Turb. Power (2003) 11-19.

[6] T. Lieuwen, V. Yang (Eds.), Combustion instabilities in gas turbine engines: Operational experience, fundamental mechanisms, and modeling, AIAA, 2006.

[7] Y. Huang, V. Yang, Dynamics and stability of lean-premixed swirl-stabilized combustion, Prog. Energy Combust. Sci. 35 (2009) 293-365.

[8] N. Docquier, S. Candel, Combution control and sensors: a review, Prog. Energy Combust. Sci. 28 (2002) 107-150.

[9] I. Emiris, J. H. Whitelaw, Control of combustion oscillations, Combust. Sci. Technol. 175 (6) (2003) 157-184.

[10] A. P. Dowling, A. S. Morgans, Feedback control of combustion oscillations, Annu. Rev. Fluid Mech. 37 (2005) 151-182.

[11] C. O. Paschereit, E. Gutmark, Active control of combustion instabilities in gas turbine burners, Proc. ASME Turbo Expo Paper no. GT2006-90186 (2006) 161-171.

[12] D. Froud, T. O'Doherty, N. Syred, Phase averaging of the precessing vortex core in a swirl burner under piloted and premixed combustion conditions, Combust. Flame 100 (1995) 407-410.

[13] N. Syred, A review of oscillation mechanisms and the role of the precessing vortex core (PVC) in swirl combustion systems, Prog. Energy Combust. Sci. 32 (2006) 93161.

[14] B. Wegner, A. Maltsev, C. Schneider, A. Sadiki, A. Dreizler, J. Janicka, Assessment of unsteady RANS in predicting swirl flow instability based on LES and experiments, Int. J. Heat Fluid Flow 25 (2004) 528536.

[15] F. Martinelli, A. Olivani, A. Coghe, Experimental analysis of the precessing vortex core in a free swirling jet, Exp. Fluids 42 (2007) 827-839.

[16] S. Wang, V. Yang, G. Hsiao, S.-Y. Hsieh, H. C. Mongia, Largeeddy simulations of gas-turbine swirl injector flow dynamics, J. Fluid Mech. 583 (2007) 99122.

[17] A. Valera-Medina, N. Syred, A. Griffiths, Visualisation of isothermal large coherent structures in a swirl burner, Combust. Flame 30 (2009) 1723-1734.

[18] A. Bourehla, F. Baillot, Appearance and stability of a laminar conical premixed flame subjected to an acoustic perturbation, Combust. Flame 114 (1998) 303-318.

[19] D. Durox, S. Ducruix, S. Candel, Experiments on collapsing cylindrical flames, Combust. Flame 125 (2001) 982-1000.

[20] S.-Y. Lee, S. Seo, J. C. Broda, S. Pal, R. J. Santoro, An experimental estimation of mean reaction rate and flame structure during combustion instability in a lean premixed gas turbine combustor, Proc. Combust. Inst. 28 (2000) 775-782.

[21] V. G. McDonell, G. S. Samuelsen, Measurement of fuel mixing and transport processes in gas turbine combustion, Meas. Sci. Technol. 11 (2000) 870-886.

[22] Y. Deguchi, M. N. abd Y. Fukuda, Y. Ichinose, Y. Endo, M. Inada, Y. Abe, S. Iwasaki, Industrial applications of temperature and species concentration monitoring using laser diagnostics, Meas. Sci. Technol. 13 (2002) R103-R115.

[23] P. Griebel, P. Siewert, P. Jansohn, Flame characteristics of turbulent lean premixed methane/air flames at high pressure: Turbulent flame speed and flame brush thickness, Proc. Combust. Inst. 31 (2007) 3083-3090.

[24] U. Stopper, M. Aigner, W. Meier, R. Sadanandan, M. Stöhr, I. K. Soo, Flow field and combustion characterization of premixed gas turbine flames by planar laser techniques, J. Eng. Gas Turb. Power 131 (2009) 021504.

[25] P. Strakey, S. D. Woodruff, T. C. Williams, R. W. Schefer, $\mathrm{OH}-$ planar fluorescence measurements of pressurized, hydrogen premixed flames in the SimVal combustor, AIAA J. 46 (2008) 1604-1613.

[26] S. Dhanuka, J. E. Temme, J. F. Driscoll, H. C. Mongia, Vortexshedding and mixing layer effects on periodic flashback in a lean 
premixed prevaporized gas turbine combustor, Proc. Combust. Inst. 32 (2009) 2901-2908.

[27] Y. Sommerer, D. Galley, T. Poinsot, S. Ducruix, F. Lacas, D. Veynante, Large-eddy simulation and experimental study of flashback and blow-off in a lean partially premixed swirled burner, J. Turbul. 5 (2004) N37.

[28] F. F. Grinstein, C. Fureby, LES studies of the flow in a swirl gas combustor, Proc. Combust. Inst. 30 (2005) 1791-1798.

[29] Y. Huang, S. Wang, V. Yang, A systematic analysis of combustion dynamics in a lean premixed swirl-stabilized combustor, AIAA J. 44 (2006) 724-740.

[30] S. Menon, N. Patel, Subgrid modeling for simulation of spray combustion in large-scale combustors, AIAA J. 44 (4) (2006) 709-723.

[31] P. Moin, S. Apte, Large-eddy simulation of realistic gas turbine combustors, AIAA J. 44 (2006) 698-708.

[32] R. Schmitt, T. Poinsot, B. Schuermans, K. P. Geigle, Largeeddy simulation and experimental study of heat transfer, nitric oxide emissions and combustion instability in a swirled turbulent high-pressure burner, J. Fluid Mech. 570 (2007) 17-46.

[33] A. Sengissen, A. Giauque, G. Staffelbach, W. K. M. Porta, P. Kaufmann, T. Poinsot, Large eddy simulation of piloting effects on turbulent swirling flames, Proc. Combust. Inst. 31 (2007) 1729-1736.

[34] H. Pitsch, LES of turbulent combustion, Annu. Rev. Fluid Mech. 28 (2006) 453-482.

[35] J. C. Oefelein, Large eddy simulation of turbulent combustion processes in propulsion and power systems, Prog. Aerosp. Sci. 42 (2006) 2-37.

[36] C. Fureby, Towards the use of large eddy simulation in engineering, Prog. Aerosp. Sci. 44 (2008) 381-396.

[37] R. Giezendanner, O. Keck, P. Weigand, W. Meier, U. Stricker, Periodic combustion instabilities in a swirl burner studied by phase-locked planar laser-induced fluorescence, Combust. Sci. Technol. 175 (2003) 721-741.

[38] R. Giezendanner, P. Weigand, X. Duan, W. Meier, U. Meier, M. Aigner, B. Lehmann, Laser based investigations of periodic combustion instabilities in a gas turbine model combustor, Combust. Sci. Technol. 127 (2005) 492-496.

[39] X. R. Duan, W. Meier, P. Weigand, B. Lehmann, Phaseresolved laser raman scattering and laser doppler velocimetry applied to periodic instabilities in a gas turbine model combustor, Appl. Phys. B 80 (2005) 389-396.

[40] P. Weigand, W. Meier, X. R. Duan, R. Giezendanner-Thoben, U. Meier, Laser diagnostic study of the mechanism of a periodic combustion instability in a gas turbine model combustor, Flow. Turb. Combust. 75 (2005) 275-292.

[41] P. Weigand, W. Meier, X. R. Duan, W. Stricker, M. Aigner, Investigations of swirl flames in a gas turbine model combustor i. Flow field, structures, temperature, and species distributions, Combust. Flame 144 (2006) 205-224.

[42] W. Meier, X. Duan, P. Weigand, Investigations of swirl flames in a gas turbine model combustor ii. Turbulence-chemistry interactions, Combust. Flame 144 (2006) 225-236.

[43] M. Stöhr, W. Meier, Coherent structures in partially premixed swirling flames, in: 12th International Symposium on Flow Visualization, Göttingen, Germany, 2006.

[44] R. Sadanandan, M. Stöhr, W. Meier, Simultaneous OH-PLIF and PIV measurements in a gas turbine model combustor, Appl. Phys. B 90 (2008) 609-618.

[45] M. Stöhr, R. Sadanandan, W. Meier, Experimental study of unsteady flame structures of an oscillating swirl flame in a gas turbine model combustor, Proc. Combust. Inst. 32 (2009) 29252932 .

[46] J. C. Broda, S. Seo, R. J. Santoro, G. Shirhattikar, V. Yang, An experimental study of combustion dynamics of a premixed swirl injector, Proc. Combust. Inst. 27 (1998) 18491856.

[47] K.-U. Schildmacher, R. Koch, H.-J. Bauer, Experimental characterization of premixed flame instabilities of a model gas turbine burner, Flow Turb. Combust. (2006) 177-197.

[48] X. Zhou, J. B. Jeffries, R. K. Hanson, G. Q. Li, E. J. Gutmark,
Wavelength-scanned tunable diode laser temperature measurements in a model gas turbine combustor, AIAA J. 45 (420-425).

49] B. Janus, A. Dreizler, J. Janicka, Experiments on swirl stabilized non-premixed natural gas flames in a model gasturbine combustor, Proc. Combust. Inst. 32 (2009) 3091-3098.

[50] I. Boxx, M. Stöhr, C. Carter, W. Meier, Temporally resolved planar measurements of transient phenomena in a partially premixed swirl flame in a gas turbine model combustor, Combust. Flame 157 (2010) 1510-1525.

[51] A. Upatnieks, J. F. Driscoll, S. L. Ceccio, C. C. Rasmussen, Liftoff of turbulent jet flames - Assessment of edge flame and other concepts using cinema-PIV, Combust. Flame 138 (3) (2004) 259-272

[52] A. M. Steinberg, J. F. Driscoll, S. L. Ceccio, Measurements of turbulent premixed flame dynamics using cinema-stereoscopic PIV, Exp. Fluids 44 (2008) 985-999.

[53] A. M. Steinberg, J. F. Driscoll, S. L. Ceccio, Temporal evolution of flame stretch due to turbulence and the hydrodynamic instability, Proc. Combust. Inst. 32 (2009) 1713-1721.

[54] A. M. Steinberg, J. F. Driscoll, Straining and wrinkling processes during turbulence-premixed flame interaction measured using temporally resolved diagnostics, Combust. Flame 156 (2009) 2285-2306.

[55] I. Boxx, C. Heeger, R. Gordon, B. Böhm, M. Aigner, A. Dreizler, W. Meier, Simultaneous three-component PIV/OH-PLIF measurements of a turbulent lifted, C3H8-Argon jet diffusion flame at $1.5 \mathrm{kHz}$ repetition rate, Proc. Combust. Inst. 32 (2009) 905-912.

[56] C. Fajardo, V. Sick, Development of a high-speed uv particle image velocimetry technique and application for measurements in internal combustion engines, Exp. Fluids 46 (2009) 43-53.

[57] A. M. Steinberg, I. Boxx, C. M. Arndt, J. H. Frank, W. Meier, Experimental study of flame-hole reignition mechanisms in a turbulent non-premixed jet flame using sustained multi-kHz PIV and crossed-plane OH PLIF, Proc. Combust. Inst. 33 (2011) In Press.

[58] S. Barbosa, P. Scouflaire, S. Ducruix, Time resolved flowfield, flame structure and acoustic characterization of a staged multiinjection burner, Proc. Combust. Inst. 32 (2009) 2965-2972.

[59] A. Prasad, Stereoscopic particle image velocimetry, Exp. Fluids 29 (2000) 103-116.

[60] I. T. Jolliffe, Principal Component Analysis, Springer, 2002.

[61] G. Berkooz, P. Holmes, J. L. Lumley, The proper orthogonal decomposition in the analysis of turbulent flows, Annu. Rev. Fluid Mech. 25 (29) (1993) 539-575.

[62] S. J. Danby, T. Echekki, Proper orthogonal decomposition analysis of autoignition simulation data of nonhomogeneous hydrogen.air mixtures, Combust. Flame 144 (2006) 126-138

[63] J. M. Donbar, J. F. Driscoll, C. D. Carter, Reaction zone structure in turbulent nonpremixed jet flames - from CH-OHPLIF images, Combust. Flame 122 (2000) 1-19.

[64] K. K. Venkataraman, L. H. Preson, D. W. Simons, B. J. Lee, J. G. Lee, D. A. Santavicca, Mechanisms of combustion instability in a lean premixed dump combustor, J. Prop. Pow. 15 (1999) 909-918

[65] J. G. Lee, D. A. Santavicca, Experimental diagnostics for the study of combustion instabilities in lean premixed combustion, J. Prop. Pow. 19 (4) (2003) 735-750.

[66] T. Lieuwen, B. Zinn, The role of equivalence ratio oscillation in driving combustion instabilities in low $\mathrm{NO}_{x}$ gas turbines, Proc. Combust. Inst. 27 (1998) 1809-1816.

[67] F. A. Richards, M. C. Janus, Control of flame oscillations with equivalence ratio modulation, J. Prop. Pow. 15 (1999) 232-240.

[68] V. Rokhlin, A. Szlam, M. Tygert, A randomized algorithm for principal component analysis, SIAM J. Martix Anal. Appl. 31 (2009) 1100-1124.

[69] C. M. Vagelopoulos, J. H. Frank, An experimental and numerical study on the adequacy of $\mathrm{CH}$ as a flame marker in premixed methane flames, Proc. Combust. Inst. 30 (2005) 241-249.

[70] A. Winkler, J. Wäsle, T. Sattelmayer, Development of a hs-lifsystem for lagrangian correlation measurement, Exp. Fluids 46 
(2008) 607-616.

[71] S. Gashi, J. Hult, K. W. Jenkins, N. C. amd S. Cant, C. F. Kaminski, Curvature and wrinkling of premixed flame kernels comparisons of OH PLIF and DNS data, Proc. Combust. Inst. 30 (2005) 809-817.

[72] M. Sweeney, S. Hochgreb, Autonomous extraction of optimal flame fronts in oh planar laser-induced fluorescence images, Appl. Optics 48 (2009) 3866-3877.

\section{A. Spatio-temporal proper orthogonal decomposi- tion analysis}

To perform the POD, the spatio-temporal data was first arranged into an $m \times n$ matrix, $A$, where each column of $A$ represented an instantaneous 2D field measurement, $u_{i}\left(x, y, t_{j}\right), j=\{1 . . n\}$. The columns were created by concatenating the rows of a planar measurement field. In order to eliminate discontinuities, the data was concatenated such that contiguous data points along a particular $y$ value remained contiguous and a subsequent $y$ value started at the same $x$ value at which the previous $y$ value finished (forming a continuous $\mathrm{S}$ pattern) [62].

POD analysis takes the matrix of $n$ observations and produces $n$ spatial eigenmodes $\left(M_{j}(x, y)\right)$, temporal coefficients $\left(a_{j}(t)\right)$, and eigenvalues $\left(\lambda_{j}\right)$ such that the eigenmodes form an orthogonal basis for $A$. The eigenvalues represent the contributions of the modes to the overall kinetic energy of the flow and the particular property of POD analysis is that it provides optimal convergence of the kinetic energy. That is, the sum of the highest $k$ eigenvalues for the POD basis is larger than for any other orthogonal basis. Assuming that the eigenvalues are sorted in descending order, the first modes represent the most dominant flow features. It is noted that the POD was performed based on the velocity data, without information about the instantaneous density field. Hence, the POD provided optimal convergence of $u_{i} u_{i}$, not the true kinetic energy $\rho u_{i} u_{i}$.

In the present work, the POD analysis was performed by approximating the singular value decomposition. That is, the $m \times n$ matrix $A$ can be represented by $A=U S V^{T}$, where $U$ is an $m \times n$ matrix, $S$ is an $n \times n$ diagonal matrix, and $V$ is an $n \times n$ matrix. It can be shown that the singular value decomposition yields the proper orthogonal decomposition [60]. The temporal evolution of the $j^{\text {th }}$ eigenmode is given by the multiplication of the $j^{t h}$ column of $U$, by the $(j, j)$ diagonal element of $S$, by the $j^{\text {th }}$ row of $V^{T}$. This results in an $m \times n$ matrix, the columns of which can be de-concatenated (reversing the concatenation procedure described above) to form the temporal history of the particular planar eigenmode. However, by considering the vector multiplication used to determine this matrix, it is apparent that the shape of the eigenmode does not change in time and is completely determined by the column of $U\left(M_{j}(x, y)=\right.$ de-concatenated $j^{t h}$ column of $\left.U\right)$. Similarly, the weighted temporal coefficient is given by $a_{j}(t)=S(j, j) \times j^{t h}$ row of $V^{T}$. The energy associated with each mode, represented by the eigenvalue, is given

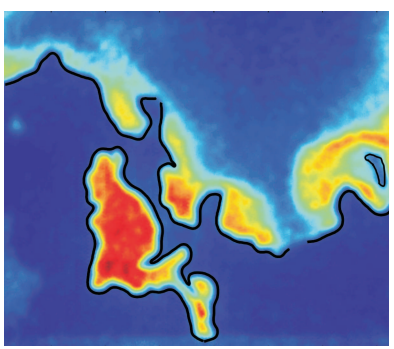
flame surface topography (a) $\mathrm{OH}$ field with mapped

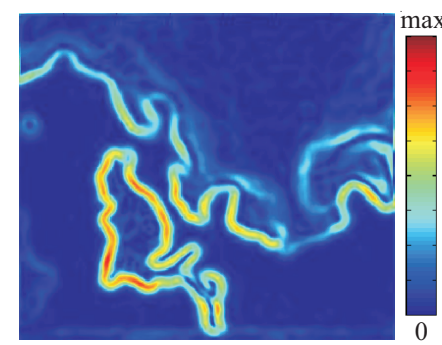

(b) Corresponding $\mathrm{OH}$ gradient magnitude

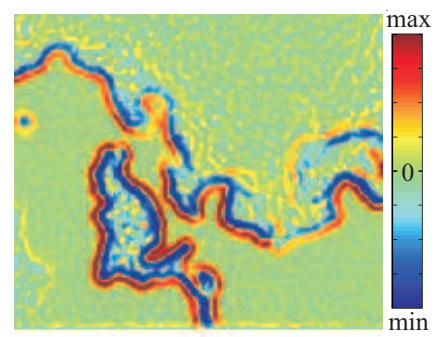

(c) Corresponding $\mathrm{OH}$ profile curvature (red is positive, blue is negative)

Figure 24: $\mathrm{OH}, \mathrm{OH}$ gradient, and $\mathrm{OH}$ curvature fields used to determine the instantaneous flame surface topography. This topography is shown as the black line in subfigure (a).

by $\lambda_{j}=S(j, j)^{2}$. However, performing a full singular value decomposition on large matrix (in this case $4350 \times$ 4000 elements) is prohibitively computationally expensive. The decomposition therefore was performed using a block Lanczos method [68].

\section{B. Identification of flame surface topography}

Quantitative detection of an instantaneous flame surface using high-speed diagnostics is a difficult task due to the low pulse energy of the laser systems. Typical combustion radicals used as flame front markers such as $\mathrm{CH}$ have very low fluorescence signal even when excited with the output of standard $10 \mathrm{~Hz}$ laser systems, which have pulse energies approximately two orders of magnitude greater than high-speed systems $[63,69]$. In kilohertz rate systems, the $\mathrm{OH}$ radical is the commonly measured combustion intermediate due to its high signal level at low laser pulse energy $[50,55,70]$. However, using $\mathrm{OH}$ as a reaction zone marker in complex flow-fields is not straightforward. Detectable amounts of $\mathrm{OH}$ exist not only in the reaction zone, but also in the high temperature products. These products mix with the non-reacted gas to produce a wide variety of $\mathrm{OH}$ fluorescence signals.

A typical $\mathrm{OH}$ field exhibiting many important features is shown in Fig. 24a. Immediately downstream of the nozzle there exists a region with no $\mathrm{OH}$, representing the unburnt reactants. Downstream from this, the $\mathrm{OH}$ signal increases suddenly at a highly corrugated and broken 
interface. The $\mathrm{OH}$ signal then decreases until a roughly conical region of uniform $\mathrm{OH}$ is reached in the products. This image also shows a thick black line, which represents the results of the flame surface topography mapping procedure outlined below.

It previously has been observed observed that the flame surface topography in such cases may be determined from the gradient of the $\mathrm{OH}$ fluorescence signal, which is shown in Fig. 24b, since $\mathrm{OH}$ exists in super-equilibrium quantities immediately downstream of the flame surface $[44,71$, 72]. The $\mathrm{OH}$ signal gradient associated with the change from reactants to products in the reaction zone was found to be much greater than the gradient as the super-equilibrium $\mathrm{OH}$ relaxed to equilibrium in the burnt gas.

Here, this idea is developed into a routine that robustly detects the flame surface topography and converts it into mathematically treatable entities. It is noted that the flame surfaces thus determined are not a direct measure of the reaction rate. A first estimate for the reactive flame surface was made by placing a global threshold on the $\mathrm{OH}$ gradient signal. It was found that there was a distinct difference between the gradients leading to super-equilibrium $\mathrm{OH}$ (i.e. at the flame front) and those associated with non-reacting interfaces between products and reactants. Hence, the identification of flame holes and recirculation was very robust and independent of the threshold level. However, over the appropriate threshold range, gradients associated with the transition from very high super-equilibrium $\mathrm{OH}$ levels at the flame to equilibrium levels in the products occasionally were detected. A secondary condition therefore was employed that eliminated detected segments that did not contact the reactants. That is, the super-equilibrium-to-equilibrium transition was distinguishable from the flame front due to the higher signal level. Finally, the somewhat broad regions of high $\mathrm{OH}$ gradient were reduced to mathematically treatable contours by simultaneously considering the $\mathrm{OH}$ profile curvature shown in Fig. 24c. The edge of the thresholded $\mathrm{OH}$ gradient region that overlapped the region of positive profile curvature was selected as the flame front. Mathematically, each flame segment in these images was then represented by a parametric curve, $f_{i}=x_{f i}\left(\vartheta_{i}\right) \hat{i}+y_{f i}\left(\vartheta_{i}\right) \hat{j}$ - The result of this procedure accurately mapped the topography of reactant to super-equilibrium $\mathrm{OH}$ transition as demonstrated in Fig. 24a. As can be seen, the routine accurately identified flame holes and isolated pockets of flame throughout the flow-field. 